DOE/ID-22244

Prepared in cooperation with the U.S. Department of Energy

\title{
Updated Procedures for Using Drill Cores and Cuttings at the Lithologic Core Storage Library, Idaho National Laboratory, Idaho
}

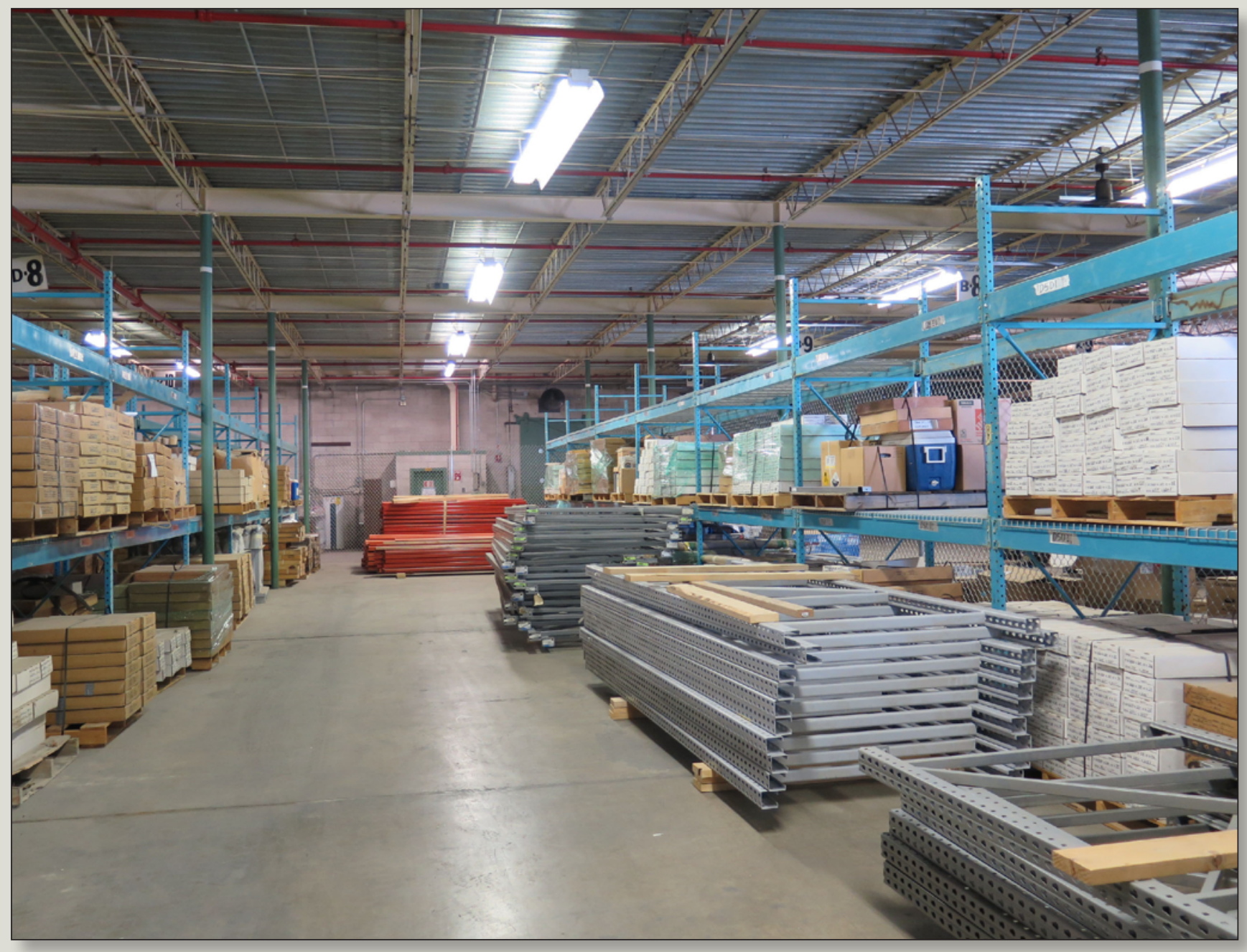

Open-File Report 2018-1001 
COVER: Lithologic Core Storage Library warehouse interior. (Photograph by Mary Hodges, U.S. Geological Survey, June 2016.) 


\section{Updated Procedures for Using Drill Cores and Cuttings at the Lithologic Core Storage Library, Idaho National Laboratory, Idaho}

By Mary K.V. Hodges, Linda C. Davis, and Roy C. Bartholomay

DOE/ID-22244

Prepared in cooperation with the U.S. Department of Energy

Open-File Report 2018-1001

U.S. Department of the Interior

U.S. Geological Survey 


\title{
U.S. Department of the Interior \\ RYAN K. ZINKE, Secretary
}

\section{U.S. Geological Survey \\ William H. Werkheiser, Deputy Director \\ exercising the authority of the Director}

\author{
U.S. Geological Survey, Reston, Virginia: 2018
}

For more information on the USGS-the Federal source for science about the Earth, its natural and living resources, natural hazards, and the environment-visit https://www.usgs.gov/ or call 1-888-ASK-USGS (1-888-275-8747).

For an overview of USGS information products, including maps, imagery, and publications, visit https:/store.usgs.gov.

Any use of trade, firm, or product names is for descriptive purposes only and does not imply endorsement by the U.S. Government.

Although this information product, for the most part, is in the public domain, it also may contain copyrighted materials as noted in the text. Permission to reproduce copyrighted items must be secured from the copyright owner.

Suggested citation:

Hodges, M.K.V., Davis, L.C., and Bartholomay, R.C., 2018, Updated procedures for using drill cores and cuttings at the Lithologic Core Storage Library, Idaho National Laboratory, Idaho: U.S. Geological Survey Open-File Report 2018-1001 (DOE/ID-22244), 48 p., https://doi.org/10.3133/ofr20181001. 


\section{Contents}

Abstract

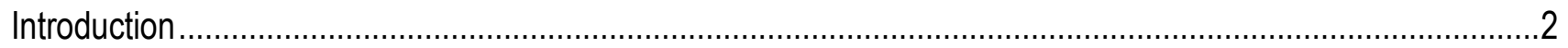

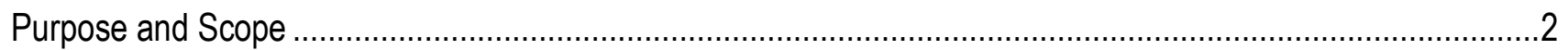

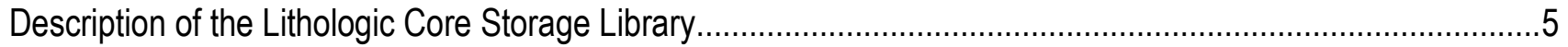

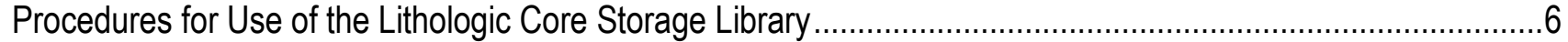

Procedure for Delivering Core to the Lithologic Core Storage Library......................................................6

Procedures for Sampling Core or Other Materials from the Lithologic Core Storage Library ..........................6

Drill Cores and Cuttings Available at the Lithologic Core Storage Library ...................................................

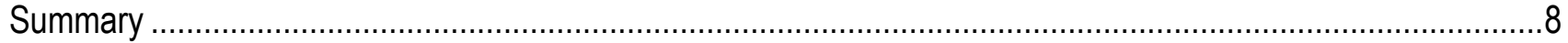

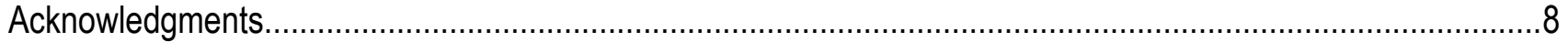

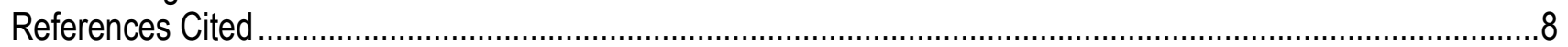

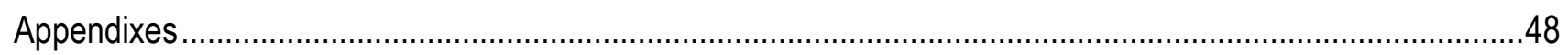

\section{Figures}

Figure 1. Locations of the Idaho National Laboratory and selected facilities and coreholes............................ 3

Figure 2. Locations of selected coreholes near the Advanced Test Reactor Complex, Idaho Nuclear

Technology and Engineering Center, and the Radioactive Waste Management Complex.....

Figure 3. Locations of buildings CF-663 and CF-674 at the Central Facilities Area, Idaho ............................ 6

\section{Tables}

Table 1. Borehole location and identifiers for core stored at the Idaho National Laboratory Lithologic

Core Storage Library

Table 2. Amount and depth below land surface of core and cuttings at the Idaho National Laboratory Lithologic Core Storage Library

Table 3. Summary of investigations on geology, paleomagnetism, and stratigraphy of the eastern

Snake River Plain and Idaho National Laboratory, Idaho 


\section{Conversion Factors}

Inch/Pound to International System of Units

\begin{tabular}{|c|c|c|}
\hline Multiply & By & To obtain \\
\hline \multicolumn{3}{|c|}{ Length } \\
\hline inch (in.) & 2.54 & centimeter $(\mathrm{cm})$ \\
\hline inch (in.) & 25.4 & millimeter $(\mathrm{mm})$ \\
\hline foot $(\mathrm{ft})$ & 0.3048 & meter $(\mathrm{m})$ \\
\hline mile (mi) & 1.609 & kilometer $(\mathrm{km})$ \\
\hline \multicolumn{3}{|c|}{ Area } \\
\hline square foot $\left(\mathrm{ft}^{2}\right)$ & 929.0 & square centimeter $\left(\mathrm{cm}^{2}\right)$ \\
\hline square foot $\left(\mathrm{ft}^{2}\right)$ & 0.09290 & square meter $\left(\mathrm{m}^{2}\right)$ \\
\hline square inch $\left(\mathrm{in}^{2}\right)$ & 6.452 & square centimeter $\left(\mathrm{cm}^{2}\right)$ \\
\hline section (640 acres or 1 square mile) & 259.0 & square hectometer $\left(\mathrm{hm}^{2}\right)$ \\
\hline square mile $\left(\mathrm{mi}^{2}\right)$ & 259.0 & hectare (ha) \\
\hline square mile $\left(\mathrm{mi}^{2}\right)$ & 2.590 & square kilometer $\left(\mathrm{km}^{2}\right)$ \\
\hline
\end{tabular}

\section{Datums}

Vertical coordinate information is referenced to the North American Vertical Datum of 1929 (NGVD 29). Horizontal coordinate information is referenced to the North American Datum of 1927 (NAD 27).

Altitude, as used in this report, refers to distance above the vertical datum. 


\title{
Updated Procedures for Using Drill Cores and Cuttings at the Lithologic Core Storage Library, Idaho National Laboratory, Idaho
}

By Mary K.V. Hodges, Linda C. Davis, and Roy C. Bartholomay

\begin{abstract}
In 1990, the U.S. Geological Survey, in cooperation with the U.S. Department of Energy Idaho Operations Office, established the Lithologic Core Storage Library at the Idaho National Laboratory (INL). The facility was established to consolidate, catalog, and permanently store nonradioactive drill cores and cuttings from subsurface investigations conducted at the INL, and to provide a location for researchers to examine, sample, and test these materials.

The facility is open by appointment to researchers for examination, sampling, and testing of cores and cuttings. This report describes the facility and cores and cuttings stored at the facility. Descriptions of cores and cuttings include the corehole names, corehole locations, and depth intervals available.

Most cores and cuttings stored at the facility were drilled at or near the INL, on the eastern Snake River Plain; however, two cores drilled on the western Snake River Plain are stored for comparative studies. Basalt, rhyolite, sedimentary interbeds, and surficial sediments compose most cores and cuttings, most of which are continuous from land surface to their total depth. The deepest continuously drilled core stored at the facility was drilled to 5,000 feet below land surface. This report describes procedures and researchers' responsibilities for access to the facility and for examination, sampling, and return of materials.
\end{abstract}




\section{Introduction}

The Idaho National Laboratory (INL) occupies about $890 \mathrm{mi}^{2}$ of the eastern Snake River Plain in southeastern Idaho (fig. 1). The INL is managed by the U.S. Department of Energy (DOE) and operated by Battelle Energy Alliance. INL facilities are used to develop peacetime nuclear-energy applications, nuclear-safety research, defense programs, and advanced energy investigations.

Since the 1950s, more than 500 test holes, auger holes, and coreholes have been drilled at the INL to characterize hydrologic and geologic conditions in the subsurface and to supply water to INL facilities. Drill cores and cuttings from some of these holes and coreholes were stored in surplus buildings and other areas at the INL and at contractor facilities in Idaho Falls, Idaho, about 50 mi east of the INL. Before 1990, no attempt had been made to consolidate, catalog, or determine the spatial distribution and physical locations of cores and cuttings available for use by researchers. Also, before 1990, many boxes containing cores were left out in the weather, rendering markings unreadable and resulting in the loss of valuable technical information for future investigations. This lack of organization caused added expenses because additional cores were sometimes drilled to obtain information that could have been obtained from existing cores.

In 1990, the INL Lithologic Core Storage Library (CSL) was established to consolidate, catalog, and permanently store nonradioactive drill cores and cuttings from investigations of the subsurface at the INL, and to provide a location for researchers to examine, sample, and test these materials. The CSL is funded by the DOE and is operated by the U.S. Geological Survey (USGS), INL Project Office. For convenience, all holes are referred to as coreholes in this report. Corehole locations for cores stored in the core library are shown in figures 1 and 2 .

\section{Purpose and Scope}

This report, prepared in cooperation with the DOE, describes the CSL and procedures for use of the CSL, and provides a list of drill cores and cuttings currently (2017) available for study at the CSL. In 2017, this list included about 73,000 ft of drill cores and several suites of drill cuttings (tables 1 and 2 , at back of report). Most of the cores and cuttings were drilled at or near the INL for studies of subsurface geohydrologic processes related to waste migration potential, geothermal potential, seismic potential, and characterization of the eastern Snake River Plain aquifer. Basalt, rhyolite, and sediment compose most of these cores and cuttings. Two cores from the western Snake River Plain are also available for comparative studies. Petrographic thin sections, primarily from cores drilled at the Radioactive Waste Management Complex (fig. 1), may be examined at the CSL. Publications containing results of analyses done on cores and cuttings are shown in table 3 (at back of report). 


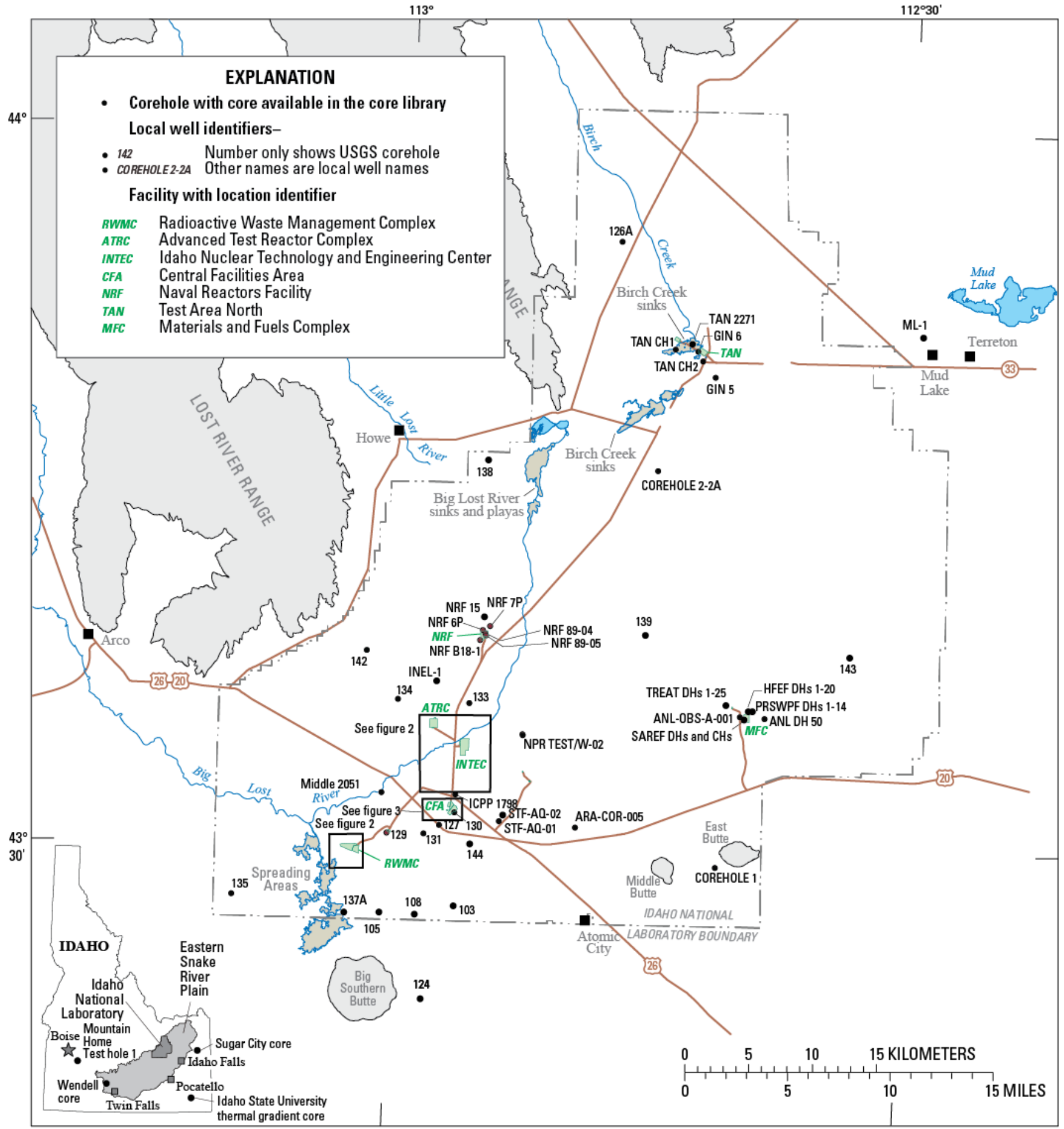

Base from U.S. Geological Survey digital data, 1:24,000 and 1:100,000

Universal Transverse Mercator projection, Zone 12

Datum is North American Datum of 1927

Figure 1. Locations of the Idaho National Laboratory and selected facilities and coreholes. 

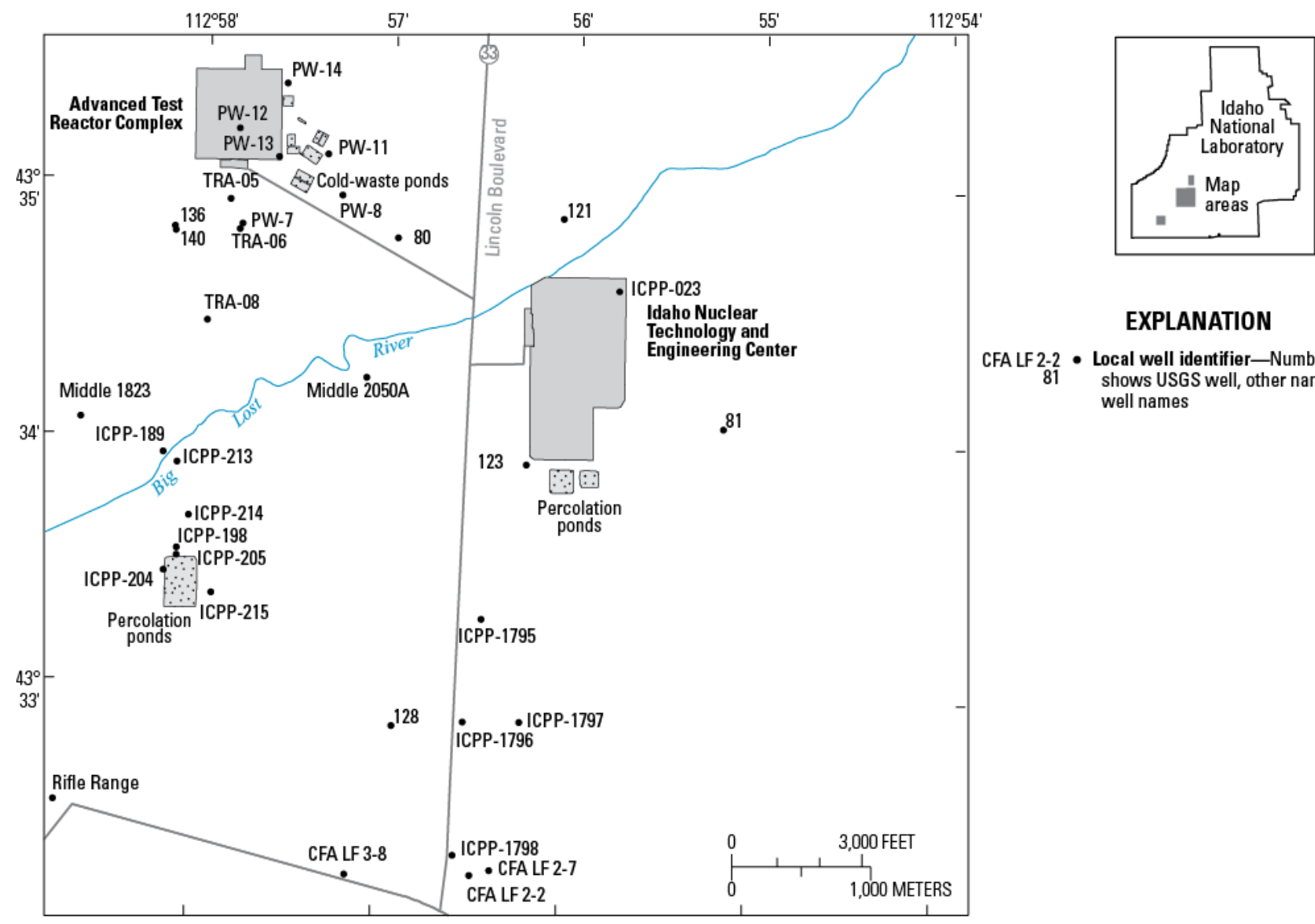

\section{EXPLANATION}

CFA LF 2-2 - Local well identifier-Numbers only

81 shows USGS well, other names are local well names

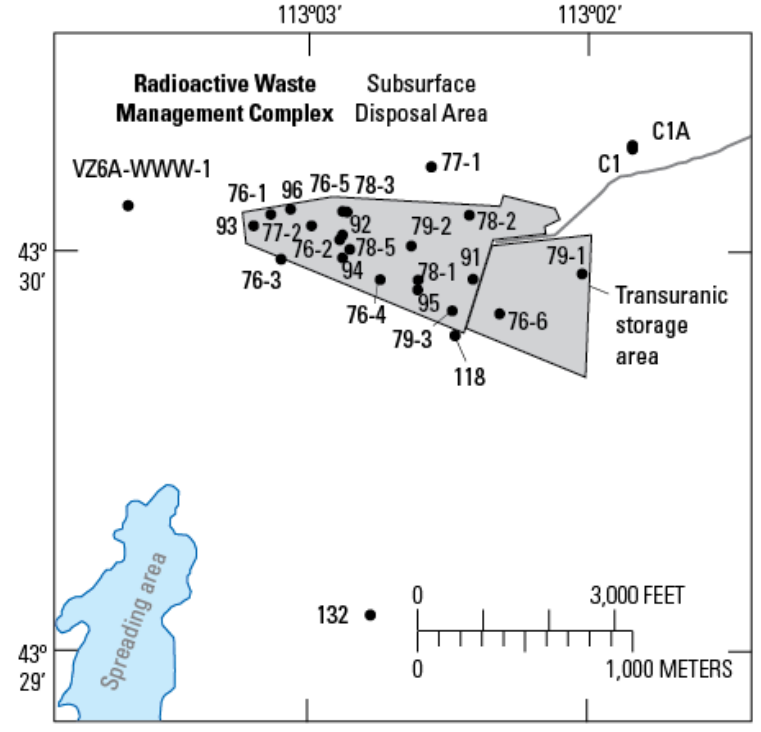

Base from U.S. Geological Survey digital data, 1:24,000 and 1:100,000

Universal Transverse Mercator projection, Zone 12

Datum is North American Datum of 1927

Figure 2. Locations of selected coreholes near the Advanced Test Reactor Complex, the Idaho Nuclear Technology and Engineering Center, and the Radioactive Waste Management Complex. 


\section{Description of the Lithologic Core Storage Library}

The CSL is located at the INL Central Facilities Area (CFA) (fig. 1), Building CFA-663 (fig. 3 ). It is a $6,163-\mathrm{ft}^{2}$ building consisting of $4,110 \mathrm{ft}^{2}$ of core storage space, $1,340 \mathrm{ft}^{2}$ of laboratory space (which includes a rock room for sample preparation), $420 \mathrm{ft}^{2}$ of office space, a $143 \mathrm{ft}^{2}$ restroom, and a $150 \mathrm{ft}^{2}$ mechanical room for heating, cooling, plumbing, and electrical systems. Additional core storage is at building CFA-674 (fig. 3), where $6,400 \mathrm{ft}^{2}$ of space is allocated for core storage.

The core-storage areas are equipped with metal racks on which pallets of cores and cuttings are stored. Most cores are packaged in waxed cardboard core boxes, each holding up to $10 \mathrm{ft}$ of core. Some cores are in 5-ft-long wooden boxes that hold 15-20 ft of core, depending on core diameter. A small forklift is used to move pallets of core on and off the racks. The core-storage area includes an examination/sampling area with several tables on which 300-400 ft of core may be laid out at a time. Equipment available for use in the examination/sampling area includes a binocular microscope, petrographic microscope, hand lenses, and an assortment of hand tools. Photographic equipment, including a digital camera, a copy stand, and accessories are also available for use.

The laboratory at the CSL is used for examining, sampling, or testing cores and cuttings. Laboratory equipment includes soil drying ovens, an oil-cooled rock saw, a water-cooled rock saw, a drill press with $1 / 2-1$ in.-diameter coring bits, petrographic microscopes, and balances, constanttemperature water baths, a mechanical sediment shaker and sieves, and an array of standard laboratory glassware and equipment. Chemicals must be provided by the users, and a Material Safety Data Sheet (MSDS) or Safety Data Sheet (SDS) must be provided to CSL personnel for each chemical brought into the laboratory. The INL contractor that operates the site for the Department of Energy, currently Battelle Energy Alliance, may have additional requirements regarding chemicals brought on the site. Some chemicals may be disallowed. The office is a work area for CSL personnel to manage the database and records related to core availability, core loaned for study, and previous investigations of the cores.

Personnel at the CSL perform many functions related to the preservation of drill cores and cuttings for scientific investigations. Chain-of-custody records are maintained and procedures followed by CSL personnel for cores for which chain-of-custody procedures were initiated at the drill site. Additionally, CSL personnel research existing information about stored cores and cuttings and maintain core-availability data. Publications containing results of analyses done on cores and cuttings are shown in table 3. 


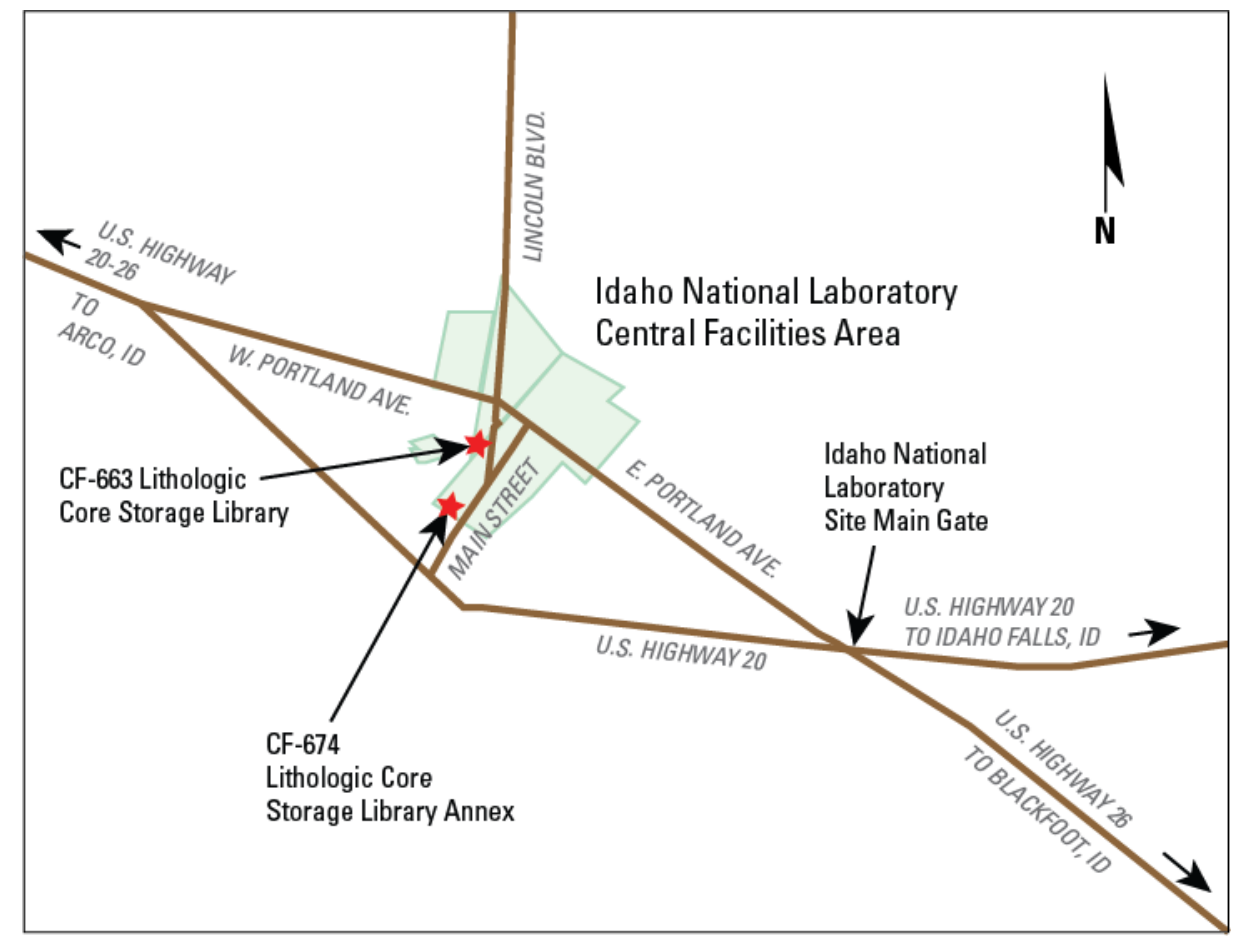

Figure 3. Locations of buildings CF-663 and CF-674 at the Central Facilities Area, Idaho.

\section{Procedures for Use of the Lithologic Core Storage Library}

\section{Procedure for Delivering Core to the Lithologic Core Storage Library}

Paper or electronic notice must be given (preferably before drilling) to the curator or to the U.S. Geological Survey INL Project Office project chief for the CSL to accept core from INL contractors or others who may wish to archive core at the CSL. Examples of the core storage notification form and core receiving logs are in appendix B. Core not drilled by USGS drill crews must be surveyed by a radiological control technician, and a radiological report must be generated (an example is shown in appendix B). A radiological report must accompany core before the CSL can accept it for storage. Chain of custody (see appendix B for example) information must also be filed with the request for core storage. Organizations and managers can choose to relinquish control of their core to the core library or to retain control, requiring permission for researchers to sample. Instructions regarding researcher access to core must be delivered electronically or in writing no later than when core is delivered to the core library.

\section{Procedures for Sampling Core or Other Materials from the Lithologic Core Storage Library}

The CSL is open to researchers for examining, sampling, or testing cores. The INL and the CSL are restricted-access areas, and permission to visit must be obtained prior to arrival at the INL. Access to the CSL is by appointment only. Appointments can be made by calling the U. S. Geological Survey Idaho National Laboratory Project Office (208) 526-2438 (administrative assistant), or by e-mailing the curator at gs-w-idif_ineel_federal_employees @usgs.gov, and putting "Core Library" in the subject line. For visitors who are United States citizens, staff can arrange access with INL security, which takes a 
minimum of 5 business days. For non-U.S. citizens, special arrangements taking 30-90 business days (depending on country of origin) must be made. Visitor identification must meet REAL ID

requirements, as specified by the U. S. Department of Homeland Security (https://www.dhs.gov/securedrivers-license-documentation, https:/www.tsa.gov/travel/security-screening/identification and https://www.dhs.gov/real-id-public-faqs). Detailed procedures for obtaining access to the CSL and permission to sample cores and cuttings can be found in the INL Lithologic Core Storage Library Standard Operating Procedures (appendix A). The CSL is open Monday through Thursday, 7:30 a.m.5:00 p.m. Arrangements for alternate days and times may be made by special request.

Researchers must provide the CSL curator with a statement of proposed research detailing the purpose and scope of the project, including a description of analyses to be done on the samples (appendix B). Researchers must specify the minimum sample size or volume needed and whether or not the analyses will destroy the samples. Requests for large volumes of samples that will be destroyed, and that may deplete an interval of core or cuttings, must be justified in writing. The curator of the CSL is responsible for obtaining sampling approval from the owner of the cores. If additional testing of samples other than that outlined in the statement of proposed research is required, written permission from the curator of the CSL or owner of the samples must be obtained prior to testing. Researchers are strongly encouraged to do their own sampling after approval has been granted. CSL personnel are available for assistance in operating machinery and recording samples borrowed. Under very limited, special circumstances, CSL personnel may be available to sample materials for researchers if the number of samples requested is fewer than 10 and if arrangements are made in advance of the time samples are needed.

One copy (preferably electronic) of published reports containing results of analyses conducted on materials furnished by the CSL, interpretations based on analyses, or unpublished data must be provided to the curator for inclusion in the records. CSL personnel can keep unpublished data confidential, if requested, but because availability of data may save other researchers from unnecessary duplication of data, release is encouraged.

All borrowed samples that may be of use to other researchers must be returned to the CSL with a description of analyses made. Portions of samples that were not used or processed also must be returned. All samples returned must be clearly marked with the number of the core hole and the depth below land surface from which they were removed. Although not required, researchers are encouraged to donate clearly marked thin sections to the CSL for archiving. Some petrographic thin sections are available for examination for some of the cores sampled in the past.

\section{Drill Cores and Cuttings Available at the Lithologic Core Storage Library}

Tables 1 and 2 are compilations of information about cores and cuttings stored at the CSL. Cores and cuttings are available for sampling after permission has been obtained from the owner of the cores or cuttings. All cores and cuttings are available for examination without permission of the owner. Data for table 1 were compiled from many sources, including Davis and others (1997), Anderson and others (1996), Bartholomay (1990a), Sehlke and others (1994), verbal communications with contractors, corehole-completion reports and diagrams, and other documentation. Because some of the data could not be verified by the authors or is incomplete, the reader is encouraged to contact the curator with corrections or additional data that may improve table 1. Cores and cuttings for which locations were not found may still be valuable for tests in which location is unimportant. Latitude and longitude were determined from maps, traditional land surveying techniques, or using the global positioning system. In this report, latitude and longitude are referenced to the North American Datum of 1927, and elevation is referenced to National Geodetic Vertical Datum of 1929. 


\section{Summary}

The Idaho National Laboratory (INL) Lithologic Core Sample Library (CSL), located at the Central Facilities Area, was established in 1990 to consolidate, catalog, and permanently store nonradioactive drill cores and cuttings, mostly from the INL subsurface. Before establishment of the CSL, cores and cuttings were stored in many locations and were not easily accessible to researchers for use. As of 2017, personnel at the CSL had identified and cataloged about 73,000 feet of drill core and several suites of cuttings which are available for examination, sampling, and testing by appointment. Some petrographic thin sections also are available for examination. In this report, drill cores and cuttings are identified by INL facility name or geographic location of the corehole, corehole name, depth intervals of cores and cuttings, latitude and longitude, and altitude. A description of the CSL equipment available for use by researchers, procedures for access, examination, and sampling and return of materials are presented.

\section{Acknowledgments}

The authors acknowledge Larry Mann for creating and sustaining the Lithologic Core Storage Library and the Department of Energy for their support of this valuable scientific resource.

\section{References Cited}

Anderson, S.R., Ackerman, D.J., Liszewski, M.J., and Freiburger, R.M., 1996, Stratigraphic data for wells at and near the Idaho National Engineering Laboratory, Idaho: U.S. Geological Survey OpenFile Report 96-248 (DOE/ID- 22127), 27 p. plus 1 diskette. [Also available at http://pubs.er.usgs.gov/usgspubs/ofr/ofr96248.]

Barraclough, J.T., Robertson, J.B., and Janzer, V.J., 1976, Hydrology of the solid waste burial ground as related to the potential migration of radionuclides, Idaho National Engineering Laboratory, with a section on drilling and sample analyses by L.G. Saindon: U.S. Geological Survey Open-File Report 76-471, $183 \mathrm{p}$.

Bartholomay, R.C., 1990a, Digitized geophysical logs for selected wells on or near the Idaho National Engineering Laboratory, Idaho: U.S. Geological Survey Open-File Report 90-366 (DOE/ ID-22088), 347 p. [Also available at http://pubs.er.usgs.gov/usgspubs/ofr/ofr90366.]

Bartholomay, R.C., 1990b, Mineralogical correlation of surficial sediment from area drainages with selected sedimentary interbeds at the Idaho National Engineering Laboratory, Idaho: U.S. Geological Survey Water-Resources Investigations Report 90-4147 (DOE/ID-22092), 18 p. [Also available at http://pubs.er.usgs.gov/usgspubs/wri/wri904147.]

Bartholomay, R.C., Knobel, L.L., and Davis, L.C., 1989, Mineralogy and grain size of surficial sediment from the Big Lost River drainage and vicinity, with chemical and physical characteristics of geologic materials from selected sites at the Idaho National Engineering Laboratory, Idaho: U.S. Geological Survey Open-File Report 89-384 (DOE/ID-22081), 74 p. [Also available at http://pubs.er.usgs.gov/usgspubs/ofr/ofr89384.]

Bestland, E.A., Link, P.K., Lanphere, M.A., and Champion, D.E., 2002, Paleoenvironments of sedimentary interbeds in the Pliocene and Quaternary Big Lost Trough (eastern Snake River Plain, Idaho), in Link, P.K., and Mink, L.L., eds., Geology, hydrogeology and environmental remediation, Idaho National Engineering and Environmental Laboratory, Eastern Snake River Plain, Idaho: Boulder, Colorado, Geological Society of America Special Paper 353, p. 27-44. 
Blair, J.J., 2002, Sedimentology and stratigraphy of sediments of the Big Lost trough subsurface from selected coreholes at the Idaho National Engineering and Environmental Laboratory: Pocatello, Idaho State University, Master's thesis, 148 p.

Champion, D.E., Dalrymple, G.B., and Kuntz, M.A., 1981, Radiometric and paleomagnetic evidence for the Emperor reversed polarity event at $0.46 \pm 0.05 \mathrm{~m}$. y. in basalt lava flows from the eastern Snake River Plain, Idaho: Geophysical Research Letters, v. 8, no. 10, p. 1,055-1,058.

Champion, D.E., Davis, L.C., Hodges, M.K.V., and Lanphere, M.A., 2013, Paleomagnetic correlation and ages of basalt flow groups in coreholes at and near the Naval Reactors Facility, Idaho National Laboratory, Idaho: U.S. Geological Survey Scientific Investigations Report 2013-5012 (DOE/ID22223), 48 p. [Also available at https://pubs.usgs.gov/sir/2013/5012/.]

Champion, D.E., and Herman, T.C., 2003, Paleomagnetism of basaltic lava flows in coreholes ICPP213, ICPP-214, ICPP-215, and USGS 128 near the vadose zone research park, Idaho Nuclear Technology and Engineering Center, Idaho National Engineering and Environmental Laboratory, Idaho: U.S. Geological Survey Open-File Report 2003-483 (DOE/ID 22189), 21 p. [Also available at https://pubs.er.usgs.gov/publication/ofr03483.]

Champion, D.E., Hodges, M.K.V., Davis, L.C., and Lanphere, M.A., 2011, Paleomagnetic correlation of surface and subsurface basaltic lava flows and flow groups in the southern part of the Idaho National Laboratory, Idaho, with paleomagnetic data tables for drill cores: U.S. Geological Survey Scientific Investigations Report 2011-5049, 34 p., 1 pl. (DOE/ID 22214), accessed February 25, 2016, at https://pubs.er.usgs.gov/publication/sir20115049.

Champion, D.E., Lanphere, M.A., Anderson, S.R., and Kuntz, M.A., 2002, Accumulation and subsidence of late Pleistocene basaltic lava flows of the eastern Snake River Plain, Idaho, in Link, P.K., and Mink, L.L., eds., Geology, hydrogeology, and environmental remediation-Idaho National Engineering and Environmental Laboratory, Eastern Snake River Plain, Idaho: Boulder, Colorado, Geological Society of America Special Paper 353, p. 175-192.

Champion, D.E., Lanphere, M.A., and Kuntz, M.A., 1988, Evidence for a new geomagnetic reversal from lava flows in Idaho-Discussion of short polarity reversals in the Brunhes and late Matuyama polarity chrons: Journal of Geophysical Research, v. 93, no. B10, p. 11,667-11,680.

Davis, L.C., Hannula, S.R., and Bowers, Beverly, 1997, Procedures for use of, and drill cores and cuttings available for study at, the Lithologic Core Storage Library, Idaho National Engineering Laboratory, Idaho: U.S. Geological Survey Open-File Report 97-124 (DOE/ID-22135), 31 p., accessed February 25, 2016, at https://pubs.er.usgs.gov/publication/ofr97124.

Doherty, D.J., 1979a, Drilling data from exploration well 2-2A, NW 1/4, sec. 15, T. 5 N., R. 31 E., Idaho National Engineering Laboratory, Butte County, Idaho: U.S. Geological Survey Open-File Report 79-851, https://pubs.er.usgs.gov/publication/ofr79851.

Doherty, D.J., 1979b, Drilling data from exploration well 1, NE 1/4, sec. 22, T. 2 N., R. 32 E., Bingham County, Idaho: U.S. Geological Survey Open-File Report 79-1225, https://pubs.er.usgs.gov/publication/ofr791225.

Doherty, D.J., McBroome, L.A., and Kuntz, M.A., 1979, Preliminary geological interpretation and lithologic log of the exploratory geothermal test well (INEL-1), Idaho National Engineering Laboratory, eastern Snake River Plain, Idaho: U.S. Geological Survey Open-File Report 79-1248, https://pubs.er.usgs.gov/publication/ofr791248.

Geist, D.J., Ellisor, R.A., Sims, E.N., and Hughes, S.S., 2002, Subsurface volcanology at Test Area North and controls on groundwater flow, in Link, P.K., and Mink, L.L., eds., Geology, hydrogeology, and environmental remediation-Idaho National Engineering and Environmental Laboratory, Eastern Snake River Plain, Idaho: Boulder, Colorado, Geological Society of America Special Paper 353, p. 45-59. 
Geslin, J.K., Link, P.K., Riesterer, J.W., Kuntz, M.A., and Fanning, C.M., 2002, Pliocene and Quaternary stratigraphic architecture and drainage systems of the Big Lost Trough, northeastern Snake River Plain, Idaho, in Link, P.K., and Mink, L.L., eds., Geology, hydrogeology, and environmental remediation-Idaho National Engineering and Environmental Laboratory, Eastern Snake River Plain, Idaho: Boulder, Colorado, Geological Society of America Special Paper 353, p. 11-26.

Gianniny, G.L., Thackray, G.D., Kaufman, D.S., Forman, S.L., Sherbondy, M.J., and Findeisen, Delda, 2002, Late Quaternary highstands in the Mud Lake and Big Lost Trough subbasins of Lake Terreton, Idaho, in Link, P.K., and Mink, L.L., eds., Geology, hydrogeology, and environmental remediationIdaho National Engineering and Environmental Laboratory, Eastern Snake River Plain, Idaho: Boulder, Colorado, Geological Society of America Special Paper 353, p. 77-90.

Grimm-Chadwick, Claire, 2004, Petrogenesis of an evolved olivine tholeiite and chemical stratigraphy of cores USGS 127, 128, and 129, Idaho National Engineering and Environmental Laboratory:

Pocatello, Idaho State University, Master's thesis, 100 p., plus apps.

Hodges, M.K.V., and Champion, D.E., 2016, Paleomagnetic correlation of basalt flows in selected coreholes near the Advanced Test Reactor Complex, the Idaho Nuclear Technology and Engineering Center, and along the southern boundary, Idaho National Laboratory, Idaho: U.S. Geological Survey Scientific Investigations Report 2016-5131 (DOE/ID-22240), 65 p., 1 pl. [Also available at https://pubs.er.usgs.gov/publication/sir20165131.]

Hodges, M.K.V., Orr, S.M., Potter, K.E., and LeMaitre, Tynan, 2012, Construction diagrams, geophysical logs, and lithologic descriptions for boreholes USGS 103, 105, 108, 131, 135, NRF-15, and NRF-16, Idaho National Laboratory, Idaho: U.S. Geological Survey Data Series 660, 34 p., accessed February 25, 2016, at https://pubs.er.usgs.gov/publication/ds660.

Hodges, M.K.V., Turrin, B.D., Champion, D.E., and Swisher, C.C., III, 2015, New argon-argon $\left({ }^{40} \mathrm{Ar} /{ }^{39} \mathrm{Ar}\right)$ radiometric age dates from selected subsurface basalt flows at the Idaho National Laboratory, Idaho: U.S. Geological Survey Scientific Investigations Report 2015-5028 (DOE/ID 22234), 25 p., accessed February 25, 2016, at https://pubs.er.usgs.gov/publication/sir20155028.

Hughes, S.S., McCurry, Michael, and Geist, D.J., 2002, Geochemical correlations and implications for the magmatic evolution of basalt flow groups at the Idaho National Engineering and Environmental Laboratory, in Link, P.K., and Mink, L.L., eds., Geology, hydrogeology, and environmental remediation-Idaho National Engineering and Environmental Laboratory, Eastern Snake River Plain, Idaho: Boulder, Colorado, Geological Society of America Special Paper 353, p. 151-173.

Kuntz, M.A., Dalrymple, G.B., Champion, D.E., and Doherty, D.J., 1980, An evaluation of potential volcanic hazards at the Radioactive Waste Management Complex, Idaho National Engineering Laboratory, Idaho: U.S. Geological Survey Open- File Report 80-388, 68 p. [Also available at http://pubs.er.usgs.gov/usgspubs/ofr/ofr80388.]

Lanphere, M.A., Champion, D.E., and Kuntz, M.A., 1993, Petrography, age, and paleomagnetism of basalt lava flows in coreholes Well 80, NRF 89-04, NRF 89-05, and ICPP 123, Idaho National Engineering Laboratory: U.S. Geological Survey Open-File Report 93-0327, 40 p. [Also available at https://pubs.er.usgs.gov/publication/ofr93327.]

Lanphere, M.A., Kuntz, M.A., and Champion, D.E., 1994, Petrography, age, and paleomagnetism of basaltic lava flows in coreholes at Test Area North (TAN), Idaho National Engineering Laboratory: U.S. Geological Survey Open-File Report 94-686, 49 p.

Mazurek, John, 2004, Genetic controls on basalt alteration within the eastern Snake River Plain aquifer system, Idaho: Idaho State University, Master's thesis, 132 p. and apps. 
Miller, M.L., 2007, Basalt stratigraphy of corehole USGS-132 with correlations and petrogenetic interpretations of the B Flow Group, Idaho National Laboratory, Idaho: Pocatello, Idaho State University, Master's thesis, 69 p. plus app. and 1 pl.

Morse, L.H., and McCurry, M.O., 2002, Genesis of alteration of Quaternary basalts within a portion of the eastern Snake River Plain aquifer, in Link, P.K., and Mink., L.L., eds., Geology, hydrogeology, and environmental remediation-Idaho National Engineering and Environmental Laboratory, Eastern Snake River Plain, Idaho: Boulder, Colorado, Geological Society of America Special Paper 353, p. 213-224.

Mudge, C.M., 2016, Properties of Pleistocene sediment in two wells in the west-central portion of the Big Lost Trough, eastern Snake River Plain, Idaho National Laboratory, Idaho: Idaho State University Master's thesis, 121 p., https://isu.app.box.com/v/Mudge-2016.

Perkins, K.S., 2003, Measurement of sedimentary interbed hydraulic properties and their hydrologic influence near the Idaho Nuclear Technology and Engineering Center at the Idaho National Engineering and Environmental Laboratory: U.S. Geological Survey Water-Resources Investigations Report 03-4048 (DOE/ID-22183), 19 p. [Also available at http://pubs.er.usgs.gov/usgspubs/wri/wri20034048.]

Perkins, K.S., and Nimmo, J.R., 2000, Measurement of hydraulic properties of the B-C interbed and their influence on contaminant transport in the unsaturated zone at the Idaho National Engineering and Environmental Laboratory, Idaho: U.S. Geological Survey Water-Resources Investigations Report 00-4073 (DOE/ID-22170), 30 p. [Also available at http://pubs.er.usgs.gov/usgspubs/wri/wri004073.]

Potter, K.E., 2010, Subsurface stratigraphy of the Arco-Big Southern Butte volcanic rift zone and implications for late pleistocene rift zone development, eastern Snake River Plain, Idaho: Pocatello, Idaho State University, Master's thesis, https://isu.app.box.com/v/Potter-2010.

Reed, M.F., Bartholomay, R.C., and Hughes, S.S., 1997, Geochemistry and stratigraphic correlation of basalt lavas beneath the Idaho Chemical Processing Plant, Idaho National Engineering Laboratory: Environmental Geology, v. 30, no. 1-2, p. 108-118.

Reed, M.F. and Bartholomay, R.C., 1994, Mineralogy of selected sedimentary interbeds at or near the Idaho National Engineering Laboratory, Idaho: U.S. Geological Survey Open- File Report 94-374 (DOE/ID-22116), 19 p. [Also available at http://pubs.er.usgs.gov/usgspubs/ofr/ofr94374.]

Rightmire, C.T., 1984, Description and hydrogeologic implications of cored sedimentary material from the 1975 drilling program at the Radioactive Waste Management Complex, Idaho: U.S. Geological Survey Water-Resources Investigations Report 84-4071 (IDO-22067), 33 p. [Also available at http://pubs.er.usgs.gov/usgspubs/wri/wri844071.]

Rightmire, C.T., and Lewis, B.D., 1987, Hydrogeology and geochemistry of the unsaturated zone, Radioactive Waste Management Complex, Idaho National Engineering Laboratory, Idaho: U.S. Geological Survey Water-Resources Investigations Report 87-4198 (DOE/ID-22073), 89 p. [Also available at http://pubs.er.usgs.gov/usgspubs/wri/wri874198.]

Scarberry, K.C., 2003, Volcanology, geochemistry, and stratigraphy of the F Basalt Flow Group, eastern Snake River Plain, Idaho: Idaho State University, Master's thesis, 139 p., 1 pl.

Sehlke, G., Davis, D.E., Smith, P.J., Jaacks, J.J., and Williams, S.J., 1994, Comprehensive well survey for the Idaho National Engineering Laboratory: U.S. Department of Energy, DOE/ID-10402, revision 3, p. 4-1-4-30.

Shervais, J.W., Vetter, S.K., and Hanan, B.B., 2006, A layered mafic sill complex beneath the eastern Snake River Plain-Evidence from cyclic geochemical variations in basalt: Geology, v. 34, no. 5, p. 365-368. 
Twining, B.V., Bartholomay, R.C, and Hodges, M.K.V., 2012, Completion summary for borehole USGS 136 near the Advanced Test Reactor Complex, Idaho National Laboratory, Idaho: U.S. Geological Survey Scientific Investigations Report 2012-5230 (DOE/ID-22220), 32 p., plus appendixes. [Also available at http://pubs.usgs.gov/sir/2012/5230/.]

Twining, B.V., Bartholomay, R.C., and Hodges, M.K.V., 2014, Completion summary for boreholes USGS 140 and USGS 141 near the Advanced Test Reactor Complex, Idaho National Laboratory, Idaho: U.S. Geological Survey Scientific Investigations Report 2014-5098 (DOE/ID-22229), 40 p., plus appendixes. [Also available at http://pubs.usgs.gov/sir/2014/5098/.]

Twining, B.V., Bartholomay, R.C., and Hodges, M.K.V., 2016, Completion summary for boreholes TAN-2271 and TAN-2272 at Test Area North, Idaho National Laboratory, Idaho: U.S. Geological Survey Scientific Investigations Report 2016-5088 (DOE/ID-22239), 37 p., plus appendixes. [Also available at http://dx.doi.org/10.3133/sir20165088.]

Twining, B.V., Hodges, M.K.V., and Orr, Stephanie, 2008, Construction diagrams, geophysical logs, and lithologic descriptions for boreholes USGS 126a, 126b, 127, 128, 129, 130, 131, 132, 133, and 134, Idaho National Laboratory, Idaho: U.S. Geological Survey Data Series Report 350 (DOE/ID22205), 27 p., and apps., accessed February 25, 2016, at http://pubs.usgs.gov/ds/350/.

Twining, B.V., Hodges, M.K.V., Schusler, Kyle, and Mudge, Christopher, 2017, Drilling, construction, geophysical log data, and lithologic log for boreholes USGS 142 and USGS 142A, Idaho National Laboratory, Idaho: U.S. Geological Survey Data Series 1058 (DOE/ID-22243), 21 p., plus appendixes, https://doi.org/10.3133/ds1058.

Wetmore, P.H., 1998, An assessment of physical volcanology and tectonics of the central eastern Snake River Plain based on the correlation of subsurface basalts at and near the Idaho National Engineering and Environmental Laboratory, Idaho: Pocatello, Idaho State University, Master's thesis https://isu.app.box.com/v/Wetmore-1998.

Winfield, K.A., 2003, Spatial variability of sedimentary interbed properties near the Idaho Nuclear Technology and Engineering Center at the Idaho National Engineering and Environmental Laboratory, Idaho: U.S. Geological Survey Water-Resources Investigations Report 03-4142 (DOE/ ID-22187), 36 p. [Also available at http://pubs.er.usgs.gov/usgspubs/wri/wri034142.] 
Table 1. Borehole location and identifiers for core stored at the Idaho National Laboratory Lithologic Core Storage Library.

[Blank cells indicate no information is available. Abbreviations: NAD 27, North American Datum of 1927; NGVD 29, National Geodetic Vertical Datum of 1929; ft, feet]

\begin{tabular}{|c|c|c|c|c|c|c|}
\hline Borehole name & Alias(es) & Site identifier & $\begin{array}{c}\text { North } \\
\text { Latitude } \\
\text { (NAD 27) }\end{array}$ & $\begin{array}{c}\text { West } \\
\text { Longitude } \\
\text { (NAD 27) }\end{array}$ & $\begin{array}{c}\text { Altitude } \\
\text { (ft above } \\
\text { sea level, } \\
\text { NGVD 29) }\end{array}$ & $\begin{array}{c}\text { Current } \\
\text { core } \\
\text { location }\end{array}$ \\
\hline \multicolumn{7}{|c|}{ Advanced Test Reactor Complex (ATR-Complex) } \\
\hline TRA 05/PZ1 & TRA-05, TRA-5 & 433453112574901 & 433453 & 1125749 & $4,926.37$ & CF-674 \\
\hline TRA 08 & & 433431112580101 & 433431 & 1125801 & $4,934.10$ & CF-674 \\
\hline PW-7 & & 433446112574602 & 433447 & 1125747 & $4,925.24$ & CF-674 \\
\hline PW-8 & & 433456112572001 & 433457 & 1125721 & $4,918.56$ & CF-674 \\
\hline PW-11 & & & 433505 & 1125722 & $4,915.60$ & CF-674 \\
\hline PW-12 & & & 433510 & 1125749 & $4,923.71$ & CF-674 \\
\hline PW-13 & & & 433505 & 1125741 & $4,923.82$ & CF-674 \\
\hline PW-14 & & & 433518 & 1125734 & $4,918.68$ & CF-674 \\
\hline USGS 80 & & 433457112570002 & 433457 & 1125700 & $4,917.00$ & CF-674 \\
\hline $\begin{array}{l}\text { TRA } 06 \text { (may not be } \\
\text { from TRA) }\end{array}$ & & & & & & CF-674 \\
\hline NRTS Hole 1 & & & & & & CF-674 \\
\hline NRTS Hole 2 & & & & & & CF-674 \\
\hline NRTS Hole 3 & & & & & & CF-674 \\
\hline NRTS Hole 4 & & & & & & CF-674 \\
\hline NRTS Hole 5 & & & & & & CF-674 \\
\hline NRTS Hole 7 & & & & & & CF-674 \\
\hline NRTS Hole 8 & & & & & & CF-674 \\
\hline NRTS Hole 9 & & & & & & CF-674 \\
\hline INEL \#1 & INEL-1, INEL 1 & 433717112563501 & 433717 & 1125636 & $4,873.29$ & CF-663 \\
\hline USGS 136 & & 433447112581501 & 433448 & 1125812 & $4,935.00$ & CF-663 \\
\hline USGS 140 & & 43344112581201 & 433441 & 1125812 & $4,936.51$ & CF-663 \\
\hline Middle 1823 & & 433418112581700 & 4334318.97 & 1125817.59 & $4,939.36$ & CF-663 \\
\hline \multicolumn{7}{|c|}{ Big Southern Butte } \\
\hline SOBU 950-1 & $950-1$ & & 432402 & 1130132 & $7,240.00$ & CF-674 \\
\hline USGS 124 & (USGS-124) & 432307112583101 & 432306 & 1125832 & $5,102.3$ & CF-674 \\
\hline USGS 103 & & 432713112560600 & 432813.57 & 1125606.23 & $5,007.42$ & CF-663 \\
\hline USGS 105 & & 432703113001700 & 432703.4 & 1130017.78 & $5,095.12$ & CF-663 \\
\hline USGS 108 & & 432658112581600 & 432658.79 & 1125823.24 & $5,031.36$ & CF-663 \\
\hline USGS 135 & & 432753113093500 & 432753.47 & 1130935.62 & $5,135.94$ & CF-663 \\
\hline USGS 137 & & 432701113025701 & 432702.33 & 1130254.01 & 5,050 & CF-663 \\
\hline USGS 137A & & 432710113025801 & 432703.07 & 1130255.62 & $5,053.81$ & CF-663 \\
\hline \multicolumn{7}{|c|}{ Box Canyon area (about 6 miles SE of Arco) } \\
\hline $11-2$ & & & & & & CF-674 \\
\hline $11-4$ & & & & & & CF-674 \\
\hline
\end{tabular}




\begin{tabular}{|c|c|c|c|c|c|c|}
\hline Borehole name & Alias(es) & Site identifier & $\begin{array}{c}\text { North } \\
\text { Latitude } \\
\text { (NAD 27) }\end{array}$ & $\begin{array}{c}\text { West } \\
\text { Longitude } \\
\text { (NAD 27) }\end{array}$ & $\begin{array}{c}\text { Altitude } \\
\text { (ft above } \\
\text { sea level, } \\
\text { NGVD 29) }\end{array}$ & $\begin{array}{l}\text { Current } \\
\text { core } \\
\text { location }\end{array}$ \\
\hline \multicolumn{7}{|c|}{ Box Canyon area (about 6 miles SE of Arco)-Continued } \\
\hline $11-5$ & & & & & & CF-674 \\
\hline $11-6$ & & & & & & CF-674 \\
\hline $\mathrm{S} 1$ & & & & & & CF-674 \\
\hline $\mathrm{S} 2$ & & & & & & CF-674 \\
\hline $\mathrm{S} 3$ & & & & & & CF-674 \\
\hline S4 & & & & & & CF-674 \\
\hline Hole \#1 & & & & & & CF-674 \\
\hline Hole \#2 & & & & & & CF-674 \\
\hline Hole \#3 & & & & & & CF-674 \\
\hline Hole \#4 & & & & & & CF-674 \\
\hline Hole \#5 & & & & & & CF-674 \\
\hline Hole \#6 & & & & & & CF-674 \\
\hline Hole \#7 & & & & & & CF-674 \\
\hline Hole \#11 & & & & & & CF-674 \\
\hline \multicolumn{7}{|c|}{ Central Facilities Area } \\
\hline USGS 127 & & 433058112572201 & 433058.28 & 1125722.04 & $4,956.44$ & CF-663 \\
\hline USGS 128 & & 433250112565601 & 433249.86 & 1125655.51 & $4,934.92$ & CF-663 \\
\hline USGS 130 & & 433130112562801 & 433130.67 & 1125628.4 & $4,927.55$ & CF-663 \\
\hline USGS 131 & & 433036112581601 & 433026.28 & 1125816.05 & $4,977.3$ & CF-663 \\
\hline USGS 144 & & 433021112552501 & 433020.58 & 1125527.98 & $4,954.22$ & CF-663 \\
\hline LF2-2 & $(\mathrm{LF} 2-02)$ & & 433218 & 1125637 & $4,932.19$ & CF-674 \\
\hline LF2-7 & (LF2-07) & & 433220 & 1125629 & $4,928.65$ & CF-674 \\
\hline LF2-7B & & & & & & CF-674 \\
\hline LF3-8 & (LF3-08) & 433218112571001 & 433218 & 1125710 & $4,940.22$ & CF-674 \\
\hline Rifle Range Well & & 433243112591101 & 433243 & 1125908 & $4,967.46$ & CF-674 \\
\hline \multicolumn{7}{|c|}{ Loss of Fluid Test Facility (LOFT) } \\
\hline LOFT-A01 & LOFT_A1 & & 435143 & 1124340 & $4,785.14$ & CF-674 \\
\hline LOFT-A02 & LOFT_A2 & & 435141 & 1124335 & $4,784.27$ & CF-674 \\
\hline LOFT-A03 & LOFT_A3 & & 435135 & 1124340 & $4,795.31$ & CF-674 \\
\hline LOFT-A04 & LOFT_A4 & & 435137 & 1124344 & $4,787.97$ & CF-674 \\
\hline LOFT-A05 & LOFT_A5 & & 435138 & 1124342 & $4,787.81$ & CF-674 \\
\hline LOFT-A06 & LOFT_A6 & & 435139 & 1124342 & $4,787.26$ & CF-674 \\
\hline LOFT-A07 & LOFT_A7 & & 435139 & 1124343 & $4,791.34$ & CF-674 \\
\hline LOFT-A08 & LOFT_A8 & & 435141 & 1124341 & $4,791.67$ & CF-674 \\
\hline LOFT-A09 & LOFT_A9 & & 435141 & 1124341 & & CF-674 \\
\hline LOFT-A10 & LOFT_A10 & & 435140 & 1124341 & 4788.4 & CF-674 \\
\hline LOFT-A11 & LOFT_A11 & & 435140 & 1124336 & 4791.42 & CF-674 \\
\hline LOFT-A12 & LOFT_A12 & & 435141 & 1124336 & 4785.76 & CF-674 \\
\hline LOFT-A13 & LOFT_A13 & & 435140 & 1124336 & 4784.54 & CF-674 \\
\hline
\end{tabular}




\begin{tabular}{|c|c|c|c|c|c|c|}
\hline Borehole name & Alias(es) & Site identifier & $\begin{array}{c}\text { North } \\
\text { Latitude } \\
\text { (NAD 27) }\end{array}$ & $\begin{array}{c}\text { West } \\
\text { Longitude } \\
\text { (NAD 27) }\end{array}$ & $\begin{array}{c}\text { Altitude } \\
\text { (ft above } \\
\text { sea level, } \\
\text { NGVD 29) }\end{array}$ & $\begin{array}{c}\text { Current } \\
\text { core } \\
\text { location }\end{array}$ \\
\hline \multicolumn{7}{|c|}{ Loss of Fluid Test Facility (LOFT)—Continued } \\
\hline LOFT-A14 & LOFT_A14 & & 435138 & 1124337 & 4790.28 & CF-674 \\
\hline LOFT-A15 & LOFT_A15 & & 435138 & 1124338 & 4788.77 & CF-674 \\
\hline LOFT-A16 & LOFT_A16 & & 435138 & 1124337 & 4791.12 & CF-674 \\
\hline LOFT-A17 & LOFT_A17 & & 435136 & 1124342 & 4782.53 & CF-674 \\
\hline LOFT-A18 & LOFT_A18 & & 435136 & 1124342 & 4785.55 & CF-674 \\
\hline LOFT-A19 & LOFT_A19 & & 435136 & 1124342 & 4791.63 & CF-674 \\
\hline LOFT-A20 & LOFT_A20 & & 435136 & 1124338 & 4794.6 & CF-674 \\
\hline \multicolumn{7}{|c|}{ Experimental Beryllium Oxide Reactor (EBOR) } \\
\hline 1 & & & & & & CF-674 \\
\hline 2 & & & & & & CF-674 \\
\hline 3 & & & & & & CF-674 \\
\hline 4 & & & & & & CF-674 \\
\hline 5 & & & & & & CF-674 \\
\hline 6 & & & & & & CF-674 \\
\hline 7 & & & & & & CF-674 \\
\hline 8 & & & & & & CF-674 \\
\hline 9 & & & & & & CF-674 \\
\hline 10 & & & & & & CF-674 \\
\hline 11 & & & & & & CF-674 \\
\hline 12 & & & & & & CF-674 \\
\hline 13 & & & & & & CF-674 \\
\hline 14 & & & & & & CF-674 \\
\hline 15 & & & & & & CF-674 \\
\hline 16 & & & & & & CF-674 \\
\hline 17 & & & & & & CF-674 \\
\hline A-1 & & & & & & CF-674 \\
\hline EBOR-01 & & & & & & CF-674 \\
\hline \multicolumn{7}{|c|}{ Idaho Nuclear Technology and Engineering Center (INTEC) } \\
\hline USGS 133 & & 433605112554301 & 433605.5 & 1125543.8 & $4,890.12$ & CF-663 \\
\hline Middle 2050 & & & & & & CF-663 \\
\hline Middle 2050A & & 433409112570500 & 433409 & 1125705 & $4,928.22$ & CF-663 \\
\hline $1 \mathrm{NW}$ & & & 433414 & 1125554 & & CF-674 \\
\hline $2 \mathrm{SE}$ & & & 433412 & 1125551 & & CF-674 \\
\hline $3 \mathrm{M}$ & & & 433413 & 1125552 & & CF-674 \\
\hline $4 \mathrm{NE}$ & & & 433414 & 1125551 & & CF-674 \\
\hline $5 \mathrm{SW}$ & & & 433412 & 1125554 & & CF-674 \\
\hline CPP_DH1_7th_Bin_Set & & & 433419 & 1125549 & & CF-674 \\
\hline CPP_DH2_7th_Bin_Set & & & 433419 & 1125549 & & CF-674 \\
\hline CPP_DH3_7th_Bin_Set & & & 433419 & 1125549 & & CF-674 \\
\hline
\end{tabular}




\begin{tabular}{|c|c|c|c|c|c|c|}
\hline Borehole name & Alias(es) & Site identifier & $\begin{array}{c}\text { North } \\
\text { Latitude } \\
\text { (NAD 27) }\end{array}$ & $\begin{array}{c}\text { West } \\
\text { Longitude } \\
\text { (NAD 27) }\end{array}$ & $\begin{array}{c}\text { Altitude } \\
\text { (ft above } \\
\text { sea level, } \\
\text { NGVD 29) }\end{array}$ & $\begin{array}{c}\text { Current } \\
\text { core } \\
\text { location }\end{array}$ \\
\hline \multicolumn{7}{|c|}{ Idaho Nuclear Technology and Engineering Center (INTEC)_Continued } \\
\hline CPP_DH4_7th_Bin_Set & & & 433418 & 1125549 & & CF-674 \\
\hline CPP_DH5_7th_Bin_Set & & & 433419 & 1125549 & & CF-674 \\
\hline CPP_DH12_7th_Bin_Set & & & & & & CF-674 \\
\hline PW-1 & & 433349112560701 & 433349 & 1125608 & $4,917.49$ & CF-674 \\
\hline PW-2 & & 433344112555601 & 433345 & 1125557 & $4,916.97$ & CF-674 \\
\hline PW-3 & & 433351112555701 & 433351 & 1125558 & $4,916.53$ & CF-674 \\
\hline PW-4 & & 433348112554901 & 433349 & 1125549 & $4,914.80$ & CF-674 \\
\hline PW-6 & & 433353112562201 & 433353 & 1125622 & $4,920.44$ & CF-674 \\
\hline СРP-01-2 & MW-16 & & 433358 & 1125602 & & CF-674 \\
\hline TF-4-1 & TF-4E, CPP_33-4-1 & & 433427 & 1125557 & & CF-674 \\
\hline TF-4-A & CPP-33-4A & & 433424 & 1125557 & & CF-674 \\
\hline TF-5-1 & TF-5, CPP-33-5L & & 433424 & 1125554 & & CF-674 \\
\hline TF-33-2-1 & TF-2, CPP-33-2 & & 433421 & 1125602 & & CF-674 \\
\hline TF-33-3-1 & TF-3, CPP33-3 & & 433424 & 1125602 & & CF-674 \\
\hline СРP 14-01 & $14-01$ & & & & & CF-674 \\
\hline СРP 14-03 & $14-03$ & & & & & CF-674 \\
\hline CPP 14-04A & $14-04 \mathrm{~A}$ & & & & & CF-674 \\
\hline CPP 14-08 & $14-08$ & & & & & CF-674 \\
\hline CPP 14-IOC & 14-IOC & & & & & CF-674 \\
\hline CРP $37-4$ & $37-4$ & & 433425 & 1125547 & & CF-674 \\
\hline USGS 81 & USGS-081, USGS-81 & 433400112551001 & 433400 & 1125510 & 4,909 & CF-674 \\
\hline USGS 121 & $\begin{array}{l}\text { ICPP-121, USGS-121 } \\
\text { ICPP-121, USGS-121, } \\
\text { A-1 through A-8 } \\
\text { (duplicate intervals } \\
\text { of USGS 121) }\end{array}$ & 433450112560301 & 433450 & 1125603 & $4,909.66$ & CF-674 \\
\hline USGS 121 & of USGS 121) & 433450112560301 & 433450 & 1125603 & $4,909.66$ & CF-663 \\
\hline USGS 123 & ICPP-123, USGS-123 & 433352112561401 & 433352 & 1125614 & $4,919.26$ & CF-663 \\
\hline ICPP-COR-A-023 & CPP-CH-AQ-01 & & 433428 & 1125541 & $4,907.88$ & CF-664 \\
\hline \multicolumn{7}{|c|}{ Idaho Falls, Idaho } \\
\hline ROB-1 & & & & & & CF-674 \\
\hline \multicolumn{7}{|c|}{ Circular Butte Landfill (CB) (proposed about 3 miles west of Mud Lake, ID) } \\
\hline $\mathrm{CB} 20$ & & & & & & CF-674 \\
\hline $\mathrm{CB} 21$ & & & & & & CF-674 \\
\hline $\mathrm{CB} 22$ & & & & & & CF-674 \\
\hline CB23 & & & & & & CF-674 \\
\hline \multicolumn{7}{|c|}{ Material and Fuels Complex (MFC) (formerly Argonne National Laboratory-West) } \\
\hline DH1 & & & 433547 & 1123923 & & CF-674 \\
\hline DH2 & & & 433547 & 1123922 & & CF-674 \\
\hline DH3 & & & 433547 & 1123922 & & CF-674 \\
\hline DH4 & & & 433547 & 1123921 & & CF-674 \\
\hline
\end{tabular}




\begin{tabular}{|c|c|c|c|c|c|}
\hline Borehole name & Site identifier & $\begin{array}{c}\text { North } \\
\text { Latitude } \\
\text { (NAD 27) }\end{array}$ & $\begin{array}{c}\text { West } \\
\text { Longitude } \\
\text { (NAD 27) }\end{array}$ & $\begin{array}{c}\text { Altitude } \\
\text { (ft above } \\
\text { sea level, } \\
\text { NGVD 29) }\end{array}$ & $\begin{array}{c}\text { Current } \\
\text { core } \\
\text { location }\end{array}$ \\
\hline \multicolumn{6}{|c|}{ Material and Fuels Complex (MFC) (formerly Argonne National Laboratory-West) } \\
\hline DH5 & & 433447 & 1123922 & & CF-674 \\
\hline DH6 & & 433547 & 1123922 & & CF-674 \\
\hline DH7 & & 433547 & 1123922 & & CF-674 \\
\hline DH8 & & 433547 & 1123921 & & CF-674 \\
\hline DH9 & & 433546 & 1123923 & & CF-674 \\
\hline DH10 & & 433546 & 1123922 & & CF-674 \\
\hline DH11 & & 433546 & 1123922 & & CF-674 \\
\hline DH12 & & 433546 & 1123922 & & CF-674 \\
\hline DH13 & & 433546 & 1123923 & & CF-674 \\
\hline DH14 & & 433546 & 1123922 & & CF-674 \\
\hline DH15 & & 433546 & 1123922 & & CF-674 \\
\hline DH16 & & 433546 & 1123921 & & CF-674 \\
\hline DH17 & & 433545 & 1123923 & & CF-674 \\
\hline DH18 & & 433545 & 1123922 & & CF-674 \\
\hline DH19 & & 433545 & 1123922 & & CF-674 \\
\hline DH20 & & 433545 & 1123921 & & CF-674 \\
\hline ANL-IWP-M1 & ANL-M1, ANL-M01 & 433555 & 1123931 & & CF-674 \\
\hline ANL-M7 & ANL-M07 & 433548 & 1123929 & & CF-674 \\
\hline ANL-M8 & ANL-M08 & 433546 & 1123929 & & CF-674 \\
\hline ANL-M9 & ANL-M09 & 433545 & 1123928 & & CF-674 \\
\hline DH1 & & 433548 & 1123920 & & CF-674 \\
\hline DH2 & & 433548 & 1123920 & & CF-674 \\
\hline DH3 & & 433548 & 1123919 & & CF-674 \\
\hline DH4 & & 433548 & 1123918 & & CF-674 \\
\hline DH5 & & 433548 & 1123919 & & CF-674 \\
\hline DH6 & & 433548 & 1123920 & & CF-674 \\
\hline DH7 & & 433548 & 1123919 & & CF-674 \\
\hline DH8 & & 433548 & 1123919 & & CF-674 \\
\hline DH9 & & 433548 & 1123918 & & CF-674 \\
\hline DH10 & & 433548 & 1123919 & & CF-674 \\
\hline DH11 & & 433547 & 1123918 & & CF-674 \\
\hline DH12 & & 433547 & 1123920 & & CF-674 \\
\hline DH13 & & 433547 & 1123918 & & CF-674 \\
\hline DH14 & & 433547 & 1123919 & & CF-674 \\
\hline $\mathrm{CH}-\mathrm{C} 7$ & & & & & CF-674 \\
\hline CH-E7.90 & & 433541 & 1123813 & & CF-674 \\
\hline CH-F11.25 & & 433539 & 1123804 & & CF-674 \\
\hline CH-G4-8.05 & & 433538 & 1123813 & & CF-674 \\
\hline $\mathrm{CH}-\mathrm{G} 12$ & & 433537 & 1123802 & & CF-674 \\
\hline
\end{tabular}




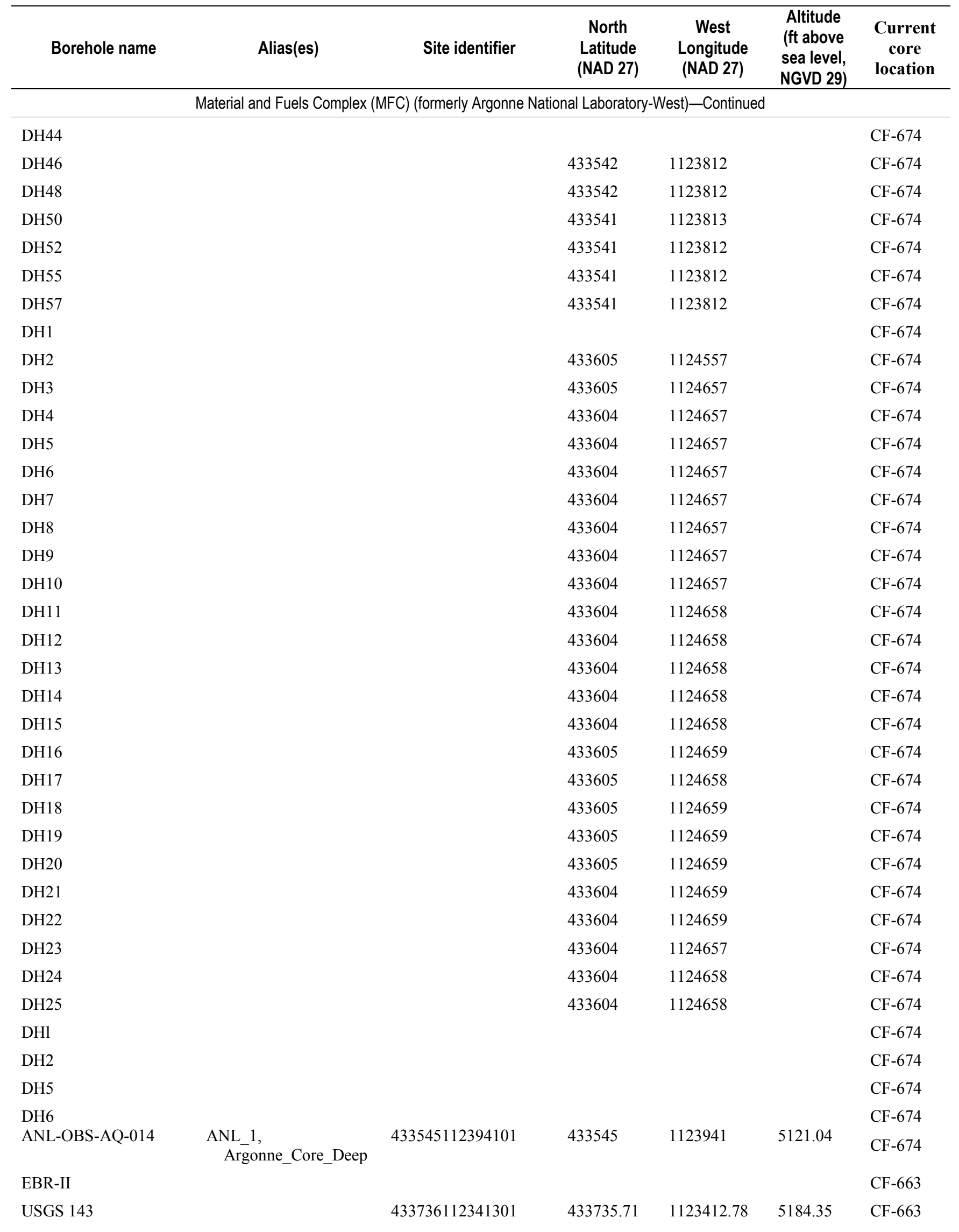




\begin{tabular}{|c|c|c|c|c|c|c|}
\hline Borehole name & Alias(es) & Site identifier & $\begin{array}{l}\text { North } \\
\text { Latitude } \\
\text { (NAD 27) }\end{array}$ & $\begin{array}{c}\text { West } \\
\text { Longitude } \\
\text { (NAD 27) }\end{array}$ & $\begin{array}{c}\text { Altitude } \\
\text { (ft above } \\
\text { sea level, } \\
\text { NGVD 29) }\end{array}$ & $\begin{array}{c}\text { Current } \\
\text { core } \\
\text { location }\end{array}$ \\
\hline \multicolumn{7}{|c|}{ Auxiliary Reactor Area (ARA) } \\
\hline ARA-COR-005 & ARA 1 & 433100112491903 & 433060 & 1124922 & 5043 & CF-674 \\
\hline ARA-MON-A-01 & ARA-MON-A-001 & & 433055 & 1124916 & & CF-674 \\
\hline ARA-MON-A-02 & ARA-MON-A-002 & & 433054 & 1124925 & & CF-674 \\
\hline ARA-MON-A-03A & ARA-MON-A-003A & & 433146 & 1124950 & & CF-674 \\
\hline ARA-MON-A-04 & ARA-MON-A-004 & & 433180 & 1124979 & & CF-674 \\
\hline STF-PIE-AQ-01 & STF & & 433111 & 1125349 & & CF-674 \\
\hline STF-PIE-AQ-02 & STF & 433127112533201 & 433127 & 1125332 & $4,937.4$ & CF-674 \\
\hline \multicolumn{7}{|c|}{ Mountain Home Air Force Base Idaho (MHAFB) } \\
\hline MHTH-1 & $\begin{array}{l}\text { Mountain_Home_Test_ } \\
\text { Hole_1 }\end{array}$ & 430319115502301 & 430319 & 1155023 & 3021 & CF-663 \\
\hline \multicolumn{7}{|c|}{ Mud Lake Landfill (ML) (about 1 mi northwest_of_Mud_Lake) } \\
\hline ML-1 & & & & & & CF-674 \\
\hline \multicolumn{7}{|c|}{ New Production Reactor (NPR) } \\
\hline A-26 & & & 433455 & 1125224 & & CF-674 \\
\hline DC-1 & & & 433453 & 1125223 & & CF-674 \\
\hline DC-2 & & & 433452 & 1125221 & & CF-674 \\
\hline DC-3 & & & 433450 & 1125220 & & CF-674 \\
\hline DC-4 & & & 433448 & 1125218 & & CF-674 \\
\hline F-1 & & & 433505 & 1125234 & & CF-674 \\
\hline $\mathrm{F}-2$ & & & 433405 & 1125237 & & CF-674 \\
\hline F-3 & & & 433404 & 1125236 & & CF-674 \\
\hline F-4 & & & 433502 & 1125235 & & CF-674 \\
\hline F-5 & & & 433504 & 1125233 & & CF-674 \\
\hline F-6 & & & 433505 & 1125231 & & CF-674 \\
\hline F-7 & & & 433506 & 1125232 & & CF-674 \\
\hline F-8 & & & 433508 & 1125233 & & CF-674 \\
\hline F-9 & & & 433507 & 1125235 & & CF-674 \\
\hline F10 & & & 433507 & 1125243 & & CF-674 \\
\hline F11 & & & 433500 & 1125236 & & CF-674 \\
\hline $\mathrm{F} 12$ & & & 433505 & 1125227 & & CF-674 \\
\hline F13 & & & 433512 & 1125234 & & CF-674 \\
\hline $\mathrm{F} 14$ & & & 433458 & 1125239 & & CF-674 \\
\hline IC-5 & & & 433455 & 1125221 & & CF-674 \\
\hline IC-7 & & & 433451 & 1125218 & & CF-674 \\
\hline IC-10 & & & 433451 & 1125223 & & CF-674 \\
\hline IC-12 & & & 433448 & 1125220 & & CF-674 \\
\hline S17 & & & 433456 & 1125222 & & CF-674 \\
\hline NPR_Test & SITE_E, NPR_E & 433449112523101 & 433449 & 1125231 & $4,933.13$ & CF-663 \\
\hline NPR & W-01, W01 & & 433451 & 1125231 & & CF-663 \\
\hline
\end{tabular}




\begin{tabular}{|c|c|c|c|c|c|c|}
\hline Borehole name & Alias(es) & Site identifier & $\begin{array}{l}\text { North } \\
\text { Latitude } \\
\text { (NAD 27) }\end{array}$ & $\begin{array}{c}\text { West } \\
\text { Longitude } \\
\text { (NAD 27) }\end{array}$ & $\begin{array}{c}\text { Altitude } \\
\text { (ft above } \\
\text { sea level, } \\
\text { NGVD 29) }\end{array}$ & $\begin{array}{c}\text { Current } \\
\text { core } \\
\text { location }\end{array}$ \\
\hline \multicolumn{7}{|c|}{ New Production Reactor (NPR)-Continued } \\
\hline NPR & W-02, W02 & 433451112523201 & 433451 & 1125232 & $4,930.29$ & CF-663 \\
\hline NPR & W-02, W02_deepened & & 433451 & 1125232 & & CF-663 \\
\hline \multicolumn{7}{|c|}{ Naval Reactors Facility (NRF) } \\
\hline NRF 6P & $\# 6 P, N R F \_6 P$ & & 433910 & 1125501 & & CF-674 \\
\hline NRF 7P & $\# 7 P, N R F \_7 P$ & & 433920 & 1125436 & & CF-674 \\
\hline NRF 89-01 & 89-01 & & & & & CF-674 \\
\hline NRF 89-02 & 89-02 & & & & & $\mathrm{CF}-674$ \\
\hline NRF 89-03 & $89-03$ & & & & & CF-674 \\
\hline NRF 89-04 & 89-04 & & 433900 & 1125451 & & CF-674 \\
\hline NRF 89-05 & $89-05$ & & 433855 & 1125450 & & CF-674 \\
\hline NRF 15 & & 433942112545001 & 433942.18 & 1125450.6 & 4841.87 & CF-663 \\
\hline NRF 16 & & 434018112545101 & 434018.28 & 1125451.4 & 4827.54 & CF-663 \\
\hline SL 1 & & & & & & CF-674 \\
\hline SL 2 & & & & & & CF-674 \\
\hline SL 3 & & & & & & CF-674 \\
\hline SL 4 & & & & & & CF-674 \\
\hline B1-2 & & & & & & CF-674 \\
\hline B2-2 & & & & & & CF-674 \\
\hline B3-2 & & & & & & CF-674 \\
\hline B4-2 & & & & & & CF-674 \\
\hline B5-2 & & & & & & CF-674 \\
\hline B6-2 & & & & & & CF-674 \\
\hline B7-2 & & & & & & CF-674 \\
\hline B8-2 & & & & & & CF-674 \\
\hline B9-2 & & & & & & CF-674 \\
\hline B10-2 & & & & & & CF-674 \\
\hline B11-2 & & & & & & CF-674 \\
\hline B12-2 & & & & & & CF-674 \\
\hline B13-2 & & & & & & CF-674 \\
\hline B14-2 & & & & & & CF-674 \\
\hline B15-2 & & & & & & CF-674 \\
\hline B16-2 & & & & & & CF-674 \\
\hline B18-2 & & & & & & CF-674 \\
\hline B1-1 & & & & & & CF-674 \\
\hline B2-1 & & & & & & CF-674 \\
\hline B3-1 & & & & & & CF-674 \\
\hline B4-1 & & & & & & CF-674 \\
\hline B5-1 & & & & & & CF-674 \\
\hline B6-1 & & & & & & CF-674 \\
\hline
\end{tabular}




\begin{tabular}{|c|c|c|c|c|c|}
\hline Borehole name & Site identifier & $\begin{array}{l}\text { North } \\
\text { Latitude } \\
\text { (NAD 27) }\end{array}$ & $\begin{array}{c}\text { West } \\
\text { Longitude } \\
\text { (NAD 27) }\end{array}$ & $\begin{array}{c}\text { Altitude } \\
\text { (ft above } \\
\text { sea level, } \\
\text { NGVD 29) }\end{array}$ & $\begin{array}{c}\text { Current } \\
\text { core } \\
\text { location }\end{array}$ \\
\hline \multicolumn{6}{|c|}{ Naval Reactors Facility (NRF) -Continued } \\
\hline B7-1 & & & & & CF-674 \\
\hline B8-1 & & & & & CF-674 \\
\hline B9-1 & & & & & CF-674 \\
\hline B10-1 & & & & & CF-674 \\
\hline B11-1 & & & & & CF-674 \\
\hline B12-1 & & & & & CF-674 \\
\hline B13-1 & & & & & CF-674 \\
\hline B14-1 & & & & & CF-674 \\
\hline B15-1 & & & & & CF-674 \\
\hline B16-1 & & & & & CF-674 \\
\hline B17-1 & & & & & CF-674 \\
\hline B18-1 & & & & & CF-663 \\
\hline SSG Prod. & & & & & CF-674 \\
\hline \multicolumn{6}{|c|}{ Idaho National Laboratory (unknown locations) } \\
\hline $\mathrm{A} 1$ & & & & & CF-674 \\
\hline A2 & & & & & CF-674 \\
\hline $\mathrm{A} 3$ & & & & & CF-674 \\
\hline A4 & & & & & CF-674 \\
\hline $5 \mathrm{~A}$ & & & & & CF-674 \\
\hline $5 \mathrm{~B}$ & & & & & CF-674 \\
\hline $5 \mathrm{C}$ & & & & & CF-674 \\
\hline $5 \mathrm{D}$ & & & & & CF-674 \\
\hline \multicolumn{6}{|c|}{ Power Burst Facility (PBF) } \\
\hline TH23 & & & & & CF-674 \\
\hline TH27 & & & & & CF-674 \\
\hline TH28 & & & & & CF-674 \\
\hline TH32 & & & & & CF-674 \\
\hline TH44 & & & & & CF-674 \\
\hline TH92 & & & & & CF-674 \\
\hline TH 100 & & & & & CF-674 \\
\hline PBF-MON-A-004 & (PBF-MW-A-004) & & & & CF-674 \\
\hline PBF-MON-A-005 & (PBF-MW-A-005 & & & & $\mathrm{CF}-674$ \\
\hline \multicolumn{6}{|c|}{ Radioactive Waste Management Area (RWMC) } \\
\hline Acid Pit-P01 & $\begin{array}{l}\text { Acid Pit PI, Acid Pit } \\
\text { Perimeter penetration } \\
\# 1\end{array}$ & 432957 & 1130241 & & CF-674 \\
\hline Acid Pit-P02 & $\begin{array}{l}\text { Acid Pit P2, Acid Pit } \\
\text { Perimeter penetration } \\
\# 2\end{array}$ & 432958 & 1130240 & & CF-674 \\
\hline Acid Pit-P03 & $\begin{array}{l}\text { Acid Pit P3, Acid Pit } \\
\text { Perimeter penetration } \\
\# 3\end{array}$ & 432958 & 1130239 & & CF-674 \\
\hline
\end{tabular}




\begin{tabular}{|c|c|c|c|c|c|c|}
\hline Borehole name & Alias(es) & Site identifier & $\begin{array}{l}\text { North } \\
\text { Latitude } \\
\text { (NAD 27) }\end{array}$ & $\begin{array}{c}\text { West } \\
\text { Longitude } \\
\text { (NAD 27) }\end{array}$ & $\begin{array}{c}\text { Altitude } \\
\text { (ft above } \\
\text { sea level, } \\
\text { NGVD 29) }\end{array}$ & $\begin{array}{c}\text { Current } \\
\text { core } \\
\text { location }\end{array}$ \\
\hline \multicolumn{7}{|c|}{ Radioactive Waste Management Area (RWMC)—Continued } \\
\hline Acid Pit-P04 & $\begin{array}{l}\text { Acid Pit P4, Acid Pit } \\
\text { Perimeter penetration } \\
\# 4\end{array}$ & & 432957 & 1130238 & & CF-674 \\
\hline Acid Pit-P05 & $\begin{array}{l}\text { Acid Pit P5, Acid Pit } \\
\text { Perimeter penetration } \\
\# 5\end{array}$ & & 432956 & 1130239 & & CF-674 \\
\hline Acid Pit-P06 & $\begin{array}{l}\text { Acid Pit P6, Acid Pit } \\
\text { Perimeter penetration } \\
\# 6\end{array}$ & & 432956 & 1130241 & & CF-674 \\
\hline B03N21 & & & & & & CF-674 \\
\hline B07N21 & & & & & & CF-674 \\
\hline B09GN11 & & & & & & CF-674 \\
\hline B10G11 & & & & & & CF-674 \\
\hline B10N21 & & & & & & CF-674 \\
\hline B14N21 & & & & & & CF-674 \\
\hline BG-76-1 & $76-1$ & & 433004 & 1130304 & & CF-674 \\
\hline BG-76-2 & $76-2$ & & 432960 & 1130253 & & CF-674 \\
\hline BG-76-3 & $76-3$ & & 432959 & 1130306 & & CF-674 \\
\hline BG-76-4 & $76-4$ & & 432955 & 1130248 & & CF-674 \\
\hline BG-76-4A & $76-4 \mathrm{~A}$ & & 432956 & 1130248 & & CF-674 \\
\hline BG-76-5 & $76-5$ & & 433004 & 1130254 & & CF-674 \\
\hline BG-76-6 & $76-6$ & & 432954 & 1130222 & & CF-674 \\
\hline BG-77-1 & $77-1$ & & 433014 & 1130242 & & CF-663 \\
\hline BG-77-2 & $77-2$ & & 433002 & 1130300 & & CF-663 \\
\hline RWMC-78-1 & $78-1$ & & 432957 & 1130237 & & CF-674 \\
\hline RWMC-78-2 & $78-2$ & & 433004 & 1130226 & & CF-674 \\
\hline RWMC-78-3 & $78-3$ & & 433004 & 1130253 & & CF-674 \\
\hline RWMC-78-5 & $78-5$ & & 433000 & 1130252 & & CF-674 \\
\hline RWMC-79-1 & $79-1$ & & 432955 & 1130203 & & CF-674 \\
\hline RWMC-79-2 & $79-2$ & & 433003 & 1130239 & & CF-674 \\
\hline RWMC-79-3 & $79-3$ & & 432949 & 1130230 & & CF-674 \\
\hline USGS 91 & USGS-091, USGS-91 & & 432957 & 1130226 & & CF-674 \\
\hline USGS 92 & USGS-092, USGS-92 & & 433001 & 1130253 & & CF-674 \\
\hline USGS 93 & $\begin{array}{l}\text { USGS-093, USGS-93 } \\
\text { USGS-093A, USGS- }\end{array}$ & & 433002 & 1130306 & & CF-674 \\
\hline USGS 93A & $93 \mathrm{~A}$ & & 433002 & 1130306 & & CF-674 \\
\hline USGS 94 & USGS-094, USGS-94 & & 432957 & 1130250 & & CF-674 \\
\hline USGS 95 & USGS-095, USGS-95 & & 432954 & 1130237 & & CF-674 \\
\hline USGS 96 & $\begin{array}{l}\text { USGS-096, USGS -96 } \\
\text { USGS-096A, USGS - }\end{array}$ & & 433004 & 1130237 & & CF-674 \\
\hline USGS 96A & $\begin{array}{l}\text { 96A } \\
\text { USGS-096B, USGS- }\end{array}$ & & 433004 & 1130259 & & CF-674 \\
\hline USGS 96B & $96 \mathrm{~B}$ & & 433004 & 1130300 & & CF-674 \\
\hline USGS 104 & USGS-104 & 432856112560801 & 432856 & 1125608 & $4,987.64$ & CF-674 \\
\hline
\end{tabular}




\begin{tabular}{|c|c|c|c|c|c|c|}
\hline Borehole name & Alias(es) & Site identifier & $\begin{array}{l}\text { North } \\
\text { Latitude } \\
\text { (NAD 27) }\end{array}$ & $\begin{array}{l}\text { West } \\
\text { Longitude } \\
\text { (NAD 27) }\end{array}$ & $\begin{array}{c}\text { Altitude } \\
\text { (ft above } \\
\text { sea level, } \\
\text { NGVD 29) }\end{array}$ & $\begin{array}{l}\text { Current } \\
\text { core } \\
\text { location }\end{array}$ \\
\hline \multicolumn{7}{|c|}{ Radioactive Waste Management Area (RWMC)—Continued } \\
\hline USGS 118 & USGS-118 & 432947113023001 & 432947 & 1130230 & $5,012.42$ & CF-674 \\
\hline RWMC-88-1D & $8801 \mathrm{D}, 88-01 \mathrm{D}$ & & 432959 & 1130240 & & CF-674 \\
\hline RWMC-88-02D & $8802 \mathrm{D}$ & & 433001 & 1130232 & & CF-674 \\
\hline RWMC-89-01D & $8901 \mathrm{D}$ & & 432959 & 1130239 & & CF-674 \\
\hline E-1 & & & & & & CF-674 \\
\hline W-1 & & & & & & CF-674 \\
\hline PIT-9-P01 & $\begin{array}{l}\text { Pit 9P1, SDA Pit } 9 \\
\text { perimeter penetration } \\
\# 1\end{array}$ & & 433002 & 1130224 & & CF-674 \\
\hline PIT-9-P02 & $\begin{array}{l}\text { Pit 9P2, SDA Pit } 9 \\
\text { perimeter penetration } \\
\# 2\end{array}$ & & 433003 & 1130223 & & CF-674 \\
\hline PIT-9-P03 & $\begin{array}{l}\text { Pit 9P3, SDA Pit } 9 \\
\text { perimeter penetration } \\
\# 3\end{array}$ & & 433005 & 1130224 & & CF-674 \\
\hline PIT-9-P04 & $\begin{array}{l}\text { Pit 9P4, SDA Pit } 9 \\
\text { perimeter penetration } \\
\# 4\end{array}$ & & 433001 & 1130224 & & CF-674 \\
\hline PIT-9-P05 & $\begin{array}{l}\text { Pit 9P5, SDA Pit } 9 \\
\text { perimeter penetration } \\
\# 5\end{array}$ & & 433001 & 1130226 & & CF-674 \\
\hline PIT-9-P06 & $\begin{array}{l}\text { Pit 9P6, SDA Pit } 9 \\
\text { perimeter penetration } \\
\# 6\end{array}$ & & 433004 & 1130225 & & CF-674 \\
\hline PIT-9-P07 & $\begin{array}{l}\text { Pit 9P7, SDA Pit } 9 \\
\text { perimeter penetration } \\
\# 7\end{array}$ & & 433003 & 1130226 & & CF-674 \\
\hline PIT-9-P08 & $\begin{array}{l}\text { Pit 9P8, SDA Pit } 9 \\
\text { perimeter penetration } \\
\# 8\end{array}$ & & 433003 & 1130223 & & CF-674 \\
\hline SIP W-05 & & & & & & CF-674 \\
\hline SIP W-06 & & & & & & CF-674 \\
\hline SIP W-09 & & & & & & CF-674 \\
\hline SIP W-11 & & & & & & CF-674 \\
\hline SIP W-13 & & & & & & CF-674 \\
\hline SIP W-17 & & & & & & CF-674 \\
\hline SIP W-17A & & & & & & CF-674 \\
\hline SIP W-18 & & & & & & CF-674 \\
\hline SIP W-24 & & & & & & CF-674 \\
\hline SIP TH-5 & & & & & & CF-674 \\
\hline SIP C-1 & & & & & & CF-674 \\
\hline DO2 & & & 433003 & 1130239 & & CF-674 \\
\hline DO6 & & & 433005 & 1130243 & & CF-674 \\
\hline DO6A & & & 433004 & 1130243 & & CF-674 \\
\hline D-10 & & & 433004 & 1130304 & & CF-674 \\
\hline D-15 & & & 432955 & 1130312 & & CF-674 \\
\hline AH-1 & & & & & & CF-674 \\
\hline
\end{tabular}




\begin{tabular}{|c|c|c|c|c|c|c|}
\hline Borehole name & Alias(es) & Site identifier & $\begin{array}{c}\text { North } \\
\text { Latitude } \\
\text { (NAD 27) }\end{array}$ & $\begin{array}{c}\text { West } \\
\text { Longitude } \\
\text { (NAD 27) }\end{array}$ & $\begin{array}{c}\text { Altitude } \\
\text { (ft above } \\
\text { sea level, } \\
\text { NGVD 29) }\end{array}$ & $\begin{array}{c}\text { Current } \\
\text { core } \\
\text { location }\end{array}$ \\
\hline \multicolumn{7}{|c|}{ Radioactive Waste Management Area (RWMC)—Continued } \\
\hline AH-1A & & & & & & CF-674 \\
\hline $\mathrm{C}-1$ & $\mathrm{C} 1$ & & 433023 & 1130204 & & CF-674 \\
\hline $\mathrm{C}-1 \mathrm{~A}$ & $\mathrm{C} 1 \mathrm{~A}, \mathrm{C} 1-\mathrm{A}$ & & 433024 & 1130204 & $5,025.21$ & CF-674 \\
\hline P-02 & & & & & & CF-674 \\
\hline WWW\#1 & WWW1, VZ6A & & 433005 & 1130333 & $5,029.70$ & CF-674 \\
\hline WWW\#2 & WWW2, vZ6 & & 433003 & 1130334 & & CF-674 \\
\hline USGS 129 & & 433036113002701 & 433036.52 & 1130027.45 & 5026.19 & CF-663 \\
\hline USGS 132 & & 432906113025001 & 432906.68 & 1130250.93 & 5028.6 & CF-663 \\
\hline \multicolumn{7}{|c|}{ Sugar City Exploration Well } \\
\hline Corehole 3 & & Sugar City, ID & & & & CF-663 \\
\hline \multicolumn{7}{|c|}{ Test Area North (TAN) } \\
\hline $\mathrm{TCH} \# 1$ & TAN CH1, TCH-1 & 435058112423401 & 435058 & 1124234 & 4780.84 & CF-674 \\
\hline TCH \#2 Piezo A & TAN CH2, TCH-2 & 435033112421701 & 435033 & 1124217 & & CF-674 \\
\hline GIN \#1 & GIN-1, GIN-01 & 434947112414301 & 434947 & 1124143 & $4,786.73$ & CF-674 \\
\hline GIN \#2 & GIN-2, GIN-02 & 434949112413401 & 434949 & 1124134 & $4,786.23$ & CF-674 \\
\hline GIN \#3 & GIN-3, GIN-03 & 434945112413101 & 434945 & 1124131 & $4,786.24$ & CF-674 \\
\hline GIN \#4 & GIN-4, GIN-04 & 434949112413601 & 434949 & 1124136 & $4,786.32$ & CF-674 \\
\hline GIN \#5 & GIN-5, GIN-05 & 434953112413301 & 434953 & 1124133 & $4,786.63$ & CF-674 \\
\hline GIN \#6 & GIN-6, GIN-06 & & 435110 & 1124348 & & CF-674 \\
\hline TAN \#27 & & & & & & CF-674 \\
\hline TAN \#28 & & & & & & CF-674 \\
\hline TAN \#29 & & & & & & CF-674 \\
\hline TAN \#30 & & & & & & CF-674 \\
\hline TAN \#30A & & & & & & CF-674 \\
\hline MW-2 & & & & & & CF-674 \\
\hline USGS 126A & & 435529112471301 & 435529 & 1124713 & 4988.69 & CF-674 \\
\hline Corehole-2A & $2-2 \mathrm{~A}$, Corehole 2-2A & 434558112444801 & 434557 & 1124449 & 4787.05 & CF-663 \\
\hline A1 & & & & & & CF-674 \\
\hline A2 & & & & & & CF-674 \\
\hline A3 & & & & & & CF-674 \\
\hline A4 & & & & & & CF-674 \\
\hline A6 & & & & & & CF-674 \\
\hline A7 & & & & & & CF-674 \\
\hline A9 & & & & & & CF-674 \\
\hline A10 & & & & & & CF-674 \\
\hline A11 & & & & & & CF-674 \\
\hline A12 & & & & & & CF-674 \\
\hline A13 & & & & & & CF-674 \\
\hline
\end{tabular}




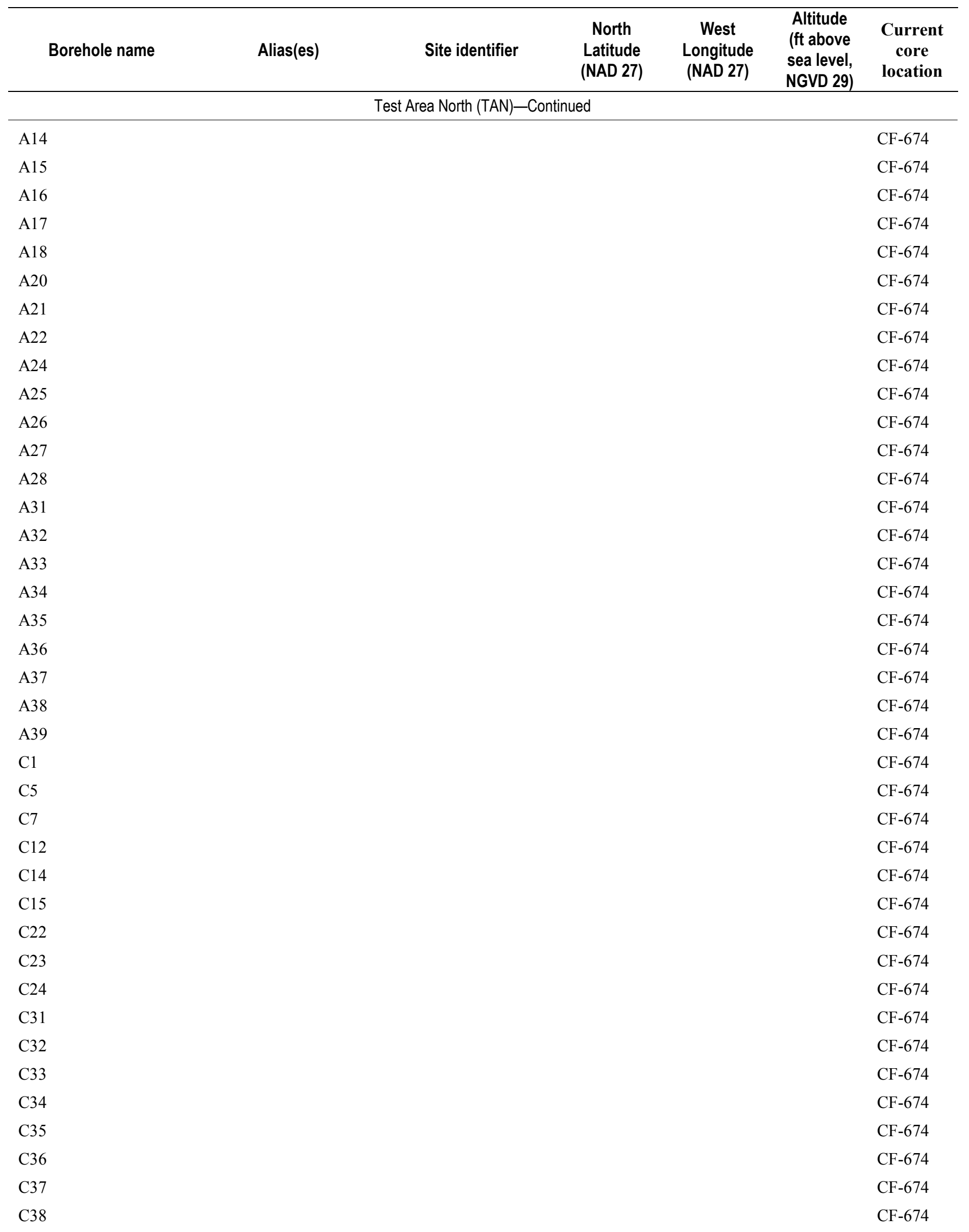




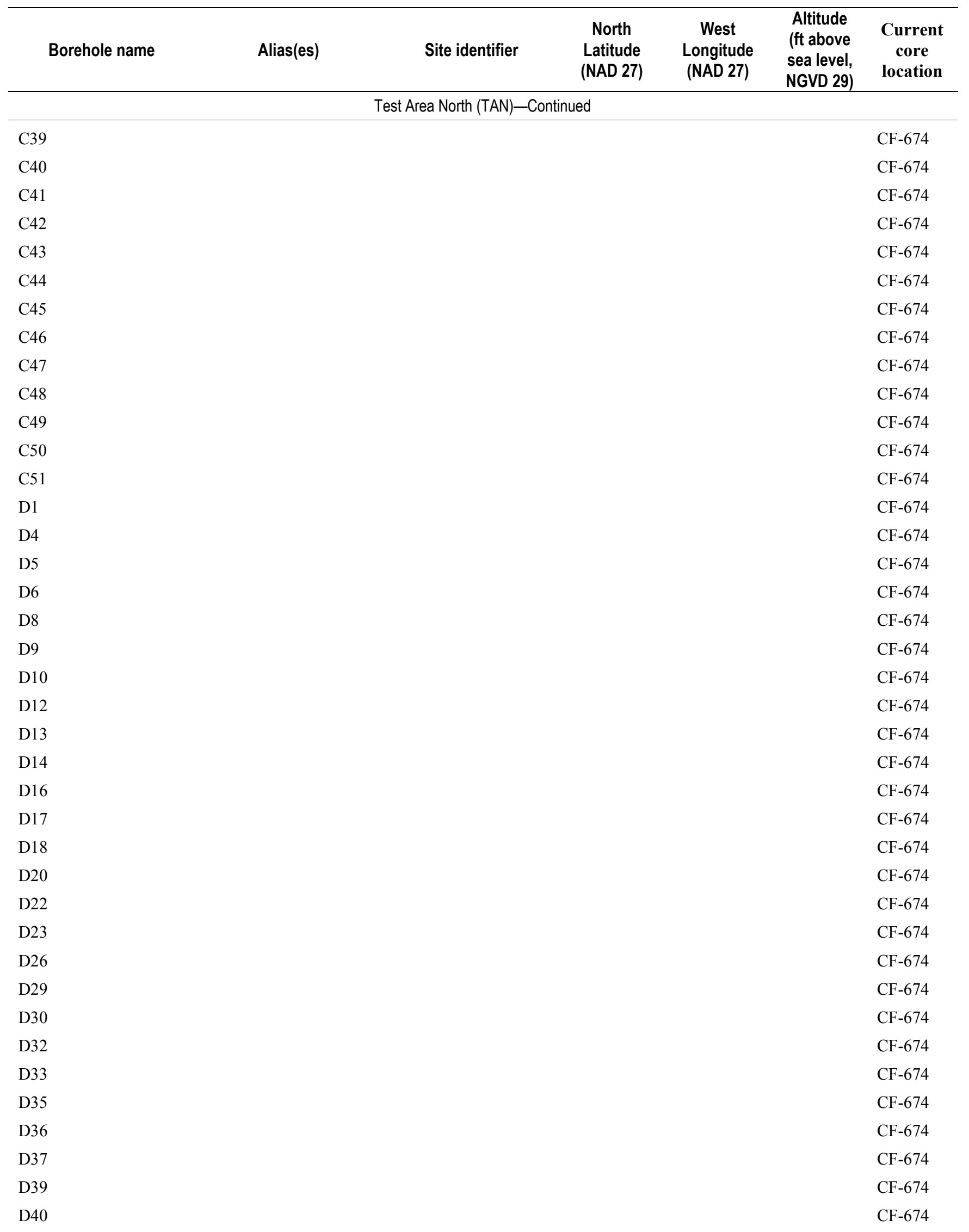




\begin{tabular}{|c|c|c|c|c|c|c|}
\hline Borehole name & Alias(es) & Site identifier & $\begin{array}{c}\text { North } \\
\text { Latitude } \\
\text { (NAD 27) }\end{array}$ & $\begin{array}{c}\text { West } \\
\text { Longitude } \\
\text { (NAD 27) }\end{array}$ & $\begin{array}{c}\text { Altitude } \\
\text { (ft above } \\
\text { sea level, } \\
\text { NGVD 29) }\end{array}$ & $\begin{array}{c}\text { Current } \\
\text { core } \\
\text { location }\end{array}$ \\
\hline \multicolumn{7}{|c|}{ Test Area North (TAN) - Continued } \\
\hline D42 & & & & & & CF-674 \\
\hline D43 & & & & & & CF-674 \\
\hline D44 & & & & & & CF-674 \\
\hline D45 & & & & & & CF-674 \\
\hline D46 & & & & & & CF-674 \\
\hline D47 & & & & & & CF-674 \\
\hline D48 & & & & & & CF-674 \\
\hline D49 & & & & & & CF-674 \\
\hline D50 & & & & & & CF-674 \\
\hline D51 & & & & & & CF-674 \\
\hline D52 & & & & & & CF-674 \\
\hline D53 & & & & & & CF-674 \\
\hline D54 & & & & & & CF-674 \\
\hline D55 & & & & & & CF-674 \\
\hline D57 & & & & & & CF-674 \\
\hline E1 & & & & & & CF-674 \\
\hline E2 & & & & & & CF-674 \\
\hline E3 & & & & & & CF-674 \\
\hline E4 & & & & & & CF-674 \\
\hline E5 & & & & & & CF-674 \\
\hline E6 & & & & & & CF-674 \\
\hline E9 & & & & & & CF-674 \\
\hline E10 & & & & & & CF-674 \\
\hline E11 & & & & & & CF-674 \\
\hline E13 & & & & & & CF-674 \\
\hline E14 & & & & & & CF-674 \\
\hline TAN 2271 & & 435053112423101 & 435053.33 & 1124231 & $4,780.98$ & CF-663 \\
\hline \multicolumn{7}{|c|}{ Wendell-RASA Test Hole } \\
\hline $\begin{array}{l}\text { Wendell-RASA Test } \\
\text { Hole }\end{array}$ & & 424955114390305 & 424955 & 424955 & $3,599.93$ & CF-663 \\
\hline \multicolumn{7}{|c|}{ Idaho National Laboratory not associated with facility } \\
\hline Exploration Well 1 & Corehole 1 & 432927112410101 & 432927 & 1124107 & 5370 & CF-674 \\
\hline CLC-3 & & & & & & CF-674 \\
\hline CLC-4A & & & & & & CF-674 \\
\hline CLC-4B & & & & & & CF-674 \\
\hline CLC-5 & & & & & & CF-674 \\
\hline CLC-6 & & & & & & CF-674 \\
\hline CLC-7 & & & & & & CF-674 \\
\hline CLC-8TR & & & & & & CF-674 \\
\hline CLC-9 & & & & & & CF-674 \\
\hline
\end{tabular}




\begin{tabular}{|c|c|c|c|c|c|c|}
\hline Borehole name & Alias(es) & Site identifier & $\begin{array}{c}\text { North } \\
\text { Latitude } \\
\text { (NAD 27) }\end{array}$ & $\begin{array}{c}\text { West } \\
\text { Longitude } \\
\text { (NAD 27) }\end{array}$ & $\begin{array}{c}\text { Altitude } \\
\text { (ft above } \\
\text { sea level, } \\
\text { NGVD 29) }\end{array}$ & $\begin{array}{c}\text { Current } \\
\text { core } \\
\text { location }\end{array}$ \\
\hline \multicolumn{7}{|c|}{ Idaho National Laboratory not associated with facility-Continued } \\
\hline CLC-10 & & & & & & CF-674 \\
\hline USGS 134 & & 433611112595801 & 433611.15 & 1125958.27 & 4968.84 & CF-663 \\
\hline USGS 138 & & 434615112553501 & 434614.95 & 1125535.05 & 4798.79 & CF-674 \\
\hline USGS 139 & & 433823112460402 & 433823 & 1124604 & 4950.68 & CF-663 \\
\hline USGS 142 & & 433837113010901 & 433837 & 1130113 & 4997 & CF-663 \\
\hline Middle 2051 & & 433217113004900 & 433216.93 & 1130049.38 & 4997.31 & CF-663 \\
\hline
\end{tabular}


Table 2. Amount and depth below land surface of core and cuttings at the Idaho National Laboratory Lithologic Core Storage Library.

[See table 1 for locations of cores. Blank cells indicate no information is available. Abbreviations: $\mathrm{ft}$, foot; mi, mile]

\begin{tabular}{|c|c|c|c|c|c|}
\hline Borehole name & Sample type & $\begin{array}{l}\text { Beginning } \\
\text { footage }\end{array}$ & $\begin{array}{l}\text { Ending } \\
\text { footage }\end{array}$ & $\begin{array}{l}\text { Total } \\
\text { footage }\end{array}$ & $\begin{array}{l}\text { Total } \\
\text { depth (ft) }\end{array}$ \\
\hline \multicolumn{6}{|c|}{ Advanced Test Reactor Complex (ATR-Complex) } \\
\hline TRA 05/PZ1 & Core & 59 & 297 & 238.0 & 297.00 \\
\hline TRA 08 (interval 1) & Core & 115 & 120 & 5.0 & \\
\hline TRA-08 (interval 2) & Core & 160 & 166.3 & 6.3 & \\
\hline TRA 08 (interval 3) & Core & 204 & 207.5 & 3.5 & 501.50 \\
\hline PW-7 & Core & 205 & 240 & 35.0 & 237.00 \\
\hline PW-8 & Core & 150 & 183 & 33.0 & 188.00 \\
\hline PW-11 & Core & 131.5 & 169.6 & 38.1 & 168.00 \\
\hline PW-12 & Core & 133.8 & 141.5 & 7.7 & 141.50 \\
\hline PW-13 & Core & 40.2 & 141.9 & 101.7 & 148.50 \\
\hline PW-14 & Core & 135.4 & 136.2 & 0.8 & 136.20 \\
\hline USGS 80 & Core & 43.9 & 203.5 & 159.6 & 204.00 \\
\hline TRA 06 & $\begin{array}{l}\text { This core may not be from } \\
\text { TRA }\end{array}$ & 24.5 & 100 & 75.5 & \\
\hline NRTS Hole 1 & Core & 41.5 & 100 & 58.5 & \\
\hline NRTS Hole 2 & Core & 53.5 & 75 & 21.5 & \\
\hline NRTS Hole 3 & Core & 59.5 & 75 & 15.5 & \\
\hline NRTS Hole 4 & Core & 45.5 & 75 & 29.5 & \\
\hline NRTS Hole 5 & Core & 39 & 100 & 61.0 & \\
\hline NRTS Hole 7 & Core & 42 & 75 & 33.0 & \\
\hline NRTS Hole 8 & Core & 34 & 75 & 41.0 & \\
\hline NRTS Hole 9 & Core & 35 & 81 & 46.0 & \\
\hline INEL \#1 & Core & 2,340 & 2,351 & 11.0 & \\
\hline INEL \#1 (con't) & Core & 2,507 & 2,515 & 8.0 & \\
\hline INEL \#1 (con't) & Core & 3,661 & 3,696 & 35.0 & \\
\hline INEL \#1 (con't) & Core & 3,768 & 3,769 & 1.0 & \\
\hline INEL \#1 (con't) & Core & 4,840 & 4,877 & 37.0 & \\
\hline INEL \#1 (con't) & Core & 9,811 & 9,812 & 1.0 & \\
\hline INEL \#1 (con't) & Core & 10,324 & 10,326 & 2.0 & $10,333.00$ \\
\hline USGS 136 & Core & 48 & 1,048 & $1,000.0$ & $1,048.00$ \\
\hline USGS 140 & Core & 0 & 546 & 500.0 & 546.00 \\
\hline Middle 1823 & Core & 500 & $1,653.1$ & $1,153.10$ & $1,653.10$ \\
\hline \multicolumn{6}{|c|}{ Big Southern Butte } \\
\hline SOBU 950-1 & Core & 13 & 1,559 & $\begin{aligned} & 1,546.00 \\
< & \text { Small piece from }\end{aligned}$ & $1,559.00$ \\
\hline USGS 124 & Core & 361 & 361 & $361 \mathrm{ft}$. & 800.00 \\
\hline USGS 103 & Core & 760 & 1,307 & 547 & $1,307.00$ \\
\hline
\end{tabular}




\begin{tabular}{|c|c|c|c|c|c|}
\hline Borehole name & Sample type & $\begin{array}{l}\text { Beginning } \\
\text { footage }\end{array}$ & $\begin{array}{l}\text { Ending } \\
\text { footage }\end{array}$ & $\begin{array}{c}\text { Total } \\
\text { footage }\end{array}$ & $\begin{array}{c}\text { Total } \\
\text { depth (ft) }\end{array}$ \\
\hline \multicolumn{6}{|c|}{ Big Southern Butte-Continued } \\
\hline USGS 105 & Core & 800 & 1,409 & 609 & $1,409.00$ \\
\hline USGS 108 & Core & 760 & 1,218 & 458 & $1,218.00$ \\
\hline USGS 135 & Core & 4 & 1,198 & 1,194 & $1,198.00$ \\
\hline USGS 137 & Core & 4 & 994 & 990 & 994.00 \\
\hline USGS 137A & Core & 984 & 1,317 & 333 & $1,317.00$ \\
\hline \multicolumn{6}{|c|}{ Box Canyon area about 6 mi southeast of Arco, ID } \\
\hline $11-2$ & Core & 2 & 61.1 & 59.1 & \\
\hline $11-4$ & Core & 1.5 & 60.1 & 58.6 & \\
\hline $11-5$ & Core & 1.2 & 73.7 & 72.5 & \\
\hline $11-6$ & Core & 2.5 & 60 & 57.5 & \\
\hline $\mathrm{S} 1$ & Core $/ 30^{\circ}$-angle hole & 1.2 & 13.6 & 12.4 & \\
\hline $\mathrm{S} 2$ & Core $/ 30^{\circ}$-angle hole & 1.3 & 17.1 & 15.8 & \\
\hline $\mathrm{S} 3$ & Core $/ 30^{\circ}$-angle hole & 1 & 37.7 & 36.7 & \\
\hline S4 & Core $/ 30^{\circ}$-angle hole & 1 & 55.4 & 54.4 & \\
\hline Hole \#1 & Core & 0 & 30.6 & 30.6 & \\
\hline Hole \#2 & Core & 0 & 29.4 & 29.4 & \\
\hline Hole \#3 & Core $/ 22^{\circ}$-angle_hole & 9.1 & 30 & 20.9 & \\
\hline Hole \#4 & Core & 0 & 30 & 30 & \\
\hline Hole \#5 & Core & 0 & 35.3 & 35.3 & \\
\hline Hole \#6 & Core & 0.5 & 29.6 & 29.1 & \\
\hline Hole \#7 & Core & 0 & 35 & 35 & \\
\hline Hole \#11 & Core/oriented corehole & 6 & 46.5 & 40.5 & \\
\hline \multicolumn{6}{|c|}{ Central Facilities Area (CFA) } \\
\hline USGS 127 & Core & 15.5 & 598 & 581 & 598.00 \\
\hline USGS 128 & Core & 23 & 768 & 745 & 768.00 \\
\hline USGS 130 & Core & 10.3 & 723 & 713 & 723.00 \\
\hline USGS 131 & Core & 10.1 & 1,239 & $1,228.90$ & $1,239.00$ \\
\hline USGS 144 & Core & 6 & 620 & 614 & 620.00 \\
\hline \multicolumn{6}{|c|}{ CFA Landfill } \\
\hline LF2-2 & Core & 641 & 646 & 5 & \\
\hline LF2-7 & Core & 629 & 661 & 32 & \\
\hline LF2-7B & Core & 50 & 70 & 20 & \\
\hline LF3-8 & Core & nidentified & & & \\
\hline \multicolumn{6}{|c|}{ CFA Rifle Range } \\
\hline Rifle Range Well & Cuttings & 0 & 440 & 440 & 620.00 \\
\hline \multicolumn{6}{|c|}{ CTF (Formerly Loss-of-Fluid Test Facility LOFT } \\
\hline LOFT-A01 & Cuttings & 0 & 25 & 25 & \\
\hline LOFT-A02 & Cuttings & 0 & 23.5 & 23.5 & \\
\hline LOFT-A03 & Cuttings & 0 & 35 & 35 & \\
\hline
\end{tabular}




\begin{tabular}{|c|c|c|c|c|c|}
\hline Borehole name & Sample type & $\begin{array}{l}\text { Beginning } \\
\text { footage }\end{array}$ & $\begin{array}{l}\text { Ending } \\
\text { footage }\end{array}$ & $\begin{array}{c}\text { Total } \\
\text { footage }\end{array}$ & $\begin{array}{c}\text { Total } \\
\text { depth (ft) }\end{array}$ \\
\hline \multicolumn{6}{|c|}{ CTF (Formerly Loss-of-Fluid Test Facility LOFT_Continued } \\
\hline LOFT-A04 & Cuttings & 0 & 23 & 23 & \\
\hline LOFT-A05 & Cuttings & 0 & 28 & 28 & \\
\hline LOFT-A06 & Cuttings & 0 & 23 & 23 & \\
\hline LOFT-A07 & Cuttings & 0 & 28.5 & 28.5 & \\
\hline LOFT-A08 & Cuttings & 0 & 25 & 25 & \\
\hline LOFT-A09 & Cuttings & 0 & 23 & 23 & \\
\hline LOFT-A10 & Cuttings & 0 & 24 & 24 & \\
\hline LOFT-All & Cuttings & 0 & 20 & 20 & \\
\hline LOFT-A12 & Cuttings & 0 & 21 & 21 & \\
\hline LOFT-A13 & Cuttings & 0 & 19.5 & 19.5 & \\
\hline LOFT-A14 & Cuttings & 0 & 29 & 29 & \\
\hline LOFT-A15 & Cuttings & 0 & 27 & 27 & \\
\hline LOFT-Al6 & Cuttings & 0 & 25 & 25 & \\
\hline LOFT-Al7 & Cuttings & 0 & 20 & 20 & \\
\hline LOFT-Al8 & Cuttings & 0 & 20 & 20 & \\
\hline LOFT-A19 & Cuttings & 0 & 27 & 27 & \\
\hline LOFT-A20 & Cuttings & 0 & 40 & 40 & \\
\hline \multicolumn{6}{|c|}{ Experimental Beryllium Oxide Reactor (EBOR) } \\
\hline 1 & Core & 25.9 & 35.9 & 10 & \\
\hline 2 & Core & 29.9 & 39.1 & 9.2 & \\
\hline 3 & Core & 27.3 & 37.3 & 10 & \\
\hline 4 & Core & 28.8 & 38.8 & 10 & \\
\hline 5 & Core & 29.8 & 39.8 & 10 & \\
\hline 6 & Core & 28.8 & 38.8 & 10 & \\
\hline 7 & Core & 27.8 & 37.8 & 10 & \\
\hline 8 & Core & 28.8 & 38.8 & 10 & \\
\hline 9 & Core & 27.8 & 37.8 & 10 & \\
\hline 10 & Core & 29 & 39 & 10 & \\
\hline 11 & Core & 28.9 & 38.9 & 10 & \\
\hline 12 & Core & 27.6 & 37.6 & 10 & \\
\hline 13 & Core & 28.6 & 38.6 & 10 & \\
\hline 14 & Core & 29.7 & 39.7 & 10 & \\
\hline 15 & Core & 31.8 & 41.8 & 10 & \\
\hline 16 & Core & 29 & 39 & 10 & \\
\hline 17 & Core & 31.1 & 41.1 & 10 & \\
\hline A-1 & Cuttings & 0 & 25 & 25 & \\
\hline EBOR-01 & Cuttings & 0 & 25 & 25 & \\
\hline \multicolumn{6}{|c|}{ Idaho Nuclear Technology and Engineering Center (INTEC) } \\
\hline USGS 133 & Core & 27.5 & 812 & 784.5 & 812.00 \\
\hline Middle 2050 & Core & 75 & 385 & 310 & 385.00 \\
\hline
\end{tabular}




\begin{tabular}{|c|c|c|c|c|c|}
\hline Borehole name & Sample type & $\begin{array}{l}\text { Beginning } \\
\text { footage }\end{array}$ & $\begin{array}{l}\text { Ending } \\
\text { footage }\end{array}$ & $\begin{array}{c}\text { Total } \\
\text { footage }\end{array}$ & $\begin{array}{c}\text { Total } \\
\text { depth (ft) }\end{array}$ \\
\hline \multicolumn{6}{|c|}{ Idaho Nuclear Technology and Engineering Center (INTEC)—Continued } \\
\hline Middle 2050A & Core & 400 & 1,379 & 979 & $1,379.00$ \\
\hline $1 \mathrm{NW}$ & Core & 47 & 61.4 & 14.4 & \\
\hline $2 \mathrm{SE}$ & Core & 43.5 & 53.1 & 9.6 & \\
\hline $3 \mathrm{M}$ & Core & 39.3 & 48.9 & 9.6 & \\
\hline $4 \mathrm{NE}$ & Core & 45 & 59.4 & 14.4 & \\
\hline $5 \mathrm{SW}$ & Core & 39.2 & 53.6 & 14.4 & \\
\hline CPP_DH1_7th_Bin_Set & Core & 44.5 & 114.5 & 70 & \\
\hline CPP_DH2_7th_Bin_Set & Core & 43 & 63 & 20 & \\
\hline CPP_DH3_7th_Bin_Set & Core & 45 & 64.4 & 19.4 & \\
\hline CPP_DH4_7th_Bin_Set & Core & 45.5 & 68.3 & 22.8 & \\
\hline CPP_DH5_7th_Bin_Set & Core & 46 & 65.4 & 19.4 & \\
\hline CPP_DH12_7th_Bin_Set & Core & 43.5 & 48.8 & 5.3 & \\
\hline PW-1 & Core & 100 & 125 & 25 & \\
\hline \multirow[t]{2}{*}{ PW-2 } & Core & 110 & 129 & 19 & \\
\hline & Core & 215 & 220 & 5 & \\
\hline PW-3 & Core & 105 & 130 & 25 & \\
\hline PW-4 & Core & 105 & 150 & 45 & \\
\hline PW-6 & Core & 100 & 125 & 25 & \\
\hline CPP-01-2 & Core & 55 & 108 & 53 & \\
\hline TF-4-1 & Core & 33.5 & 124.1 & 90.6 & \\
\hline TF-4-A & Core & 42.2 & 112.7 & 70.5 & \\
\hline TF-5-1 & Core & 35.5 & 131.5 & 96 & \\
\hline TF-33-2-1 & Core & 41.3 & 114.8 & 73.5 & \\
\hline TF-33-3-1 & Core & 45.8 & 126.4 & 80.6 & \\
\hline CPP 14-01 & Core & 34.6 & 54.6 & 20 & \\
\hline CPP 14-03 & Core & 35.5 & 55.5 & 20 & \\
\hline СРP 14-04A & Core & 38.7 & 55 & 16.3 & \\
\hline CPP 14-08 & Core & 30.5 & 55 & 24.5 & \\
\hline CPP 14-IOC & Core & 45.3 & 55.3 & 10 & \\
\hline СРР 37-4 & Core & 34.1 & 105.7 & 71.6 & \\
\hline USGS 81 & Core & 8.5 & 107 & 98.5 & 107.00 \\
\hline USGS 121 & Core & 0 & 745.8 & 745.8 & 745.80 \\
\hline USGS 121 & Core & 40 & 68.2 & 28.2 & \\
\hline USGS 123 & Core & 0 & 741.7 & 741.7 & 744.20 \\
\hline ICPP-COR-A-023 & Core & 40.7 & 738.6 & 697.9 & 740.30 \\
\hline \multicolumn{6}{|c|}{ Idaho Falls, Idaho about 50 mi east of the CFA } \\
\hline ROB-1 & Core & 9.5 & 53.1 & 43.6 & \\
\hline \multicolumn{6}{|c|}{ Circular Butte Landfill (CB) (proposed) (about 3 mi west of Mud Lake, ID) } \\
\hline CB20 & Core & 0 & 185 & 185 & \\
\hline CB21 & Core & 0 & 155 & 155 & \\
\hline
\end{tabular}




\begin{tabular}{|c|c|c|c|c|c|}
\hline Borehole name & Sample type & $\begin{array}{l}\text { Beginning } \\
\text { footage }\end{array}$ & $\begin{array}{l}\text { Ending } \\
\text { footage }\end{array}$ & $\begin{array}{c}\text { Total } \\
\text { footage }\end{array}$ & $\begin{array}{c}\text { Total } \\
\text { depth (ft) }\end{array}$ \\
\hline \multicolumn{6}{|c|}{ Circular Butte Landfill (CB) (proposed) (about 3 mi west of Mud Lake, ID)_Continued } \\
\hline CB22 & Core & 0 & 160 & 160 & \\
\hline \multirow[t]{2}{*}{ CB23 } & Core & 0 & 163 & 163 & \\
\hline & Core & 95 & 153 & 58 & \\
\hline \multicolumn{6}{|c|}{ Material and Fuels Complex (formerly Argonne National Laboratory West) } \\
\hline DH1 & Core & 8 & 80 & 72 & \\
\hline DH2 & Core & 13.5 & 33.5 & 20 & \\
\hline DH3 & Core & 15 & 35 & 20 & \\
\hline DH4 & Core & 18.5 & 51.1 & 32.6 & \\
\hline DH5 & Core & 15 & 53 & 38 & \\
\hline DH6 & Core & 11 & 55 & 44 & \\
\hline DH7 & Core & 18.6 & 80 & 61.4 & \\
\hline DH8 & Core & 15.5 & 49.5 & 34 & \\
\hline DH9 & Core & 15.5 & 45 & 29.5 & \\
\hline DH10 & Core & 17 & 54.5 & 37.5 & \\
\hline DH11 & Core & 12 & 55 & 43 & \\
\hline DH12 & Core & 14.8 & 34.8 & 20 & \\
\hline DH13 & Core & 13 & 35.5 & 22.5 & \\
\hline DH14 & Core & 8 & 79 & 71 & \\
\hline DH15 & Core & 14.5 & 46 & 31.5 & \\
\hline DH16 & Core & 13 & 35.5 & 22.5 & \\
\hline DH17 & Core & 13.5 & 51 & 37.5 & \\
\hline DH18 & Core & 10 & 35 & 25 & \\
\hline DH19 & Core & 15 & 35 & 20 & \\
\hline DH20 & Core & 5 & 49 & 44 & \\
\hline ANL-IWP-M1 & Core & 7 & 54 & 47 & \\
\hline ANL-M7 & Core & 46.5 & 60 & 13.5 & \\
\hline ANL-M8 & Core & 36 & 54.5 & 18.5 & \\
\hline ANL-M9 & Core & 37 & 55 & 18 & \\
\hline DH1 & Core & 4.5 & 37.5 & 33 & \\
\hline $\mathrm{DH} 2$ & Core & 4.5 & 62.5 & 58 & \\
\hline DH3 & Core & 15 & 35 & 20 & \\
\hline DH4 & Core & 17 & 92 & 75 & \\
\hline DH5 & Core & 11.5 & 52.5 & 41 & \\
\hline DH6 & Core & 15.2 & 72 & 56.8 & \\
\hline DH7 & Core & 10 & 76.5 & 66.5 & \\
\hline DH8 & Core & 10 & 90 & 80 & \\
\hline DH9 & Core & 15 & 35 & 20 & \\
\hline DH10 & Core & 12 & 52.5 & 40.5 & \\
\hline DH11 & Core & 14.6 & 68.5 & 53.9 & \\
\hline DH12 & Core & 8.5 & 77 & 68.5 & \\
\hline
\end{tabular}




\begin{tabular}{|c|c|c|c|c|c|}
\hline Borehole name & Sample type & $\begin{array}{l}\text { Beginning } \\
\text { footage }\end{array}$ & $\begin{array}{l}\text { Ending } \\
\text { footage }\end{array}$ & $\begin{array}{c}\text { Total } \\
\text { footage }\end{array}$ & $\begin{array}{c}\text { Total } \\
\text { depth (ft) }\end{array}$ \\
\hline \multicolumn{6}{|c|}{ Material and Fuels Complex (formerly Argonne National Laboratory West)-Continued } \\
\hline DH13 & Core & 10 & 52 & 42 & \\
\hline DH14 & Core & 12.5 & 35 & 22.5 & \\
\hline $\mathrm{CH}-\mathrm{C} 7$ & Core & 8 & 125 & 117 & \\
\hline CH-E7.90 & Core & 8 & 128 & 120 & \\
\hline CH-F11.25 & Core & 8 & 127.9 & 119.9 & \\
\hline CH-G4-8.05 & Core & 13 & 125 & 112 & \\
\hline $\mathrm{CH}-\mathrm{G} 12$ & Core & 9.5 & 125 & 115.5 & \\
\hline DH44 & Core & 5.2 & 125.7 & 120.5 & \\
\hline DH46 & Core & 6.8 & 125.3 & 118.5 & \\
\hline DH48 & Core & 7.5 & 27 & 19.5 & \\
\hline DH50 & Core & 8.1 & 250.5 & 242.4 & \\
\hline DH52 & Core & 7.5 & 50 & 42.5 & \\
\hline DH55 & Core & 6.7 & 125.2 & 118.5 & \\
\hline DH57 & Core & 7.6 & 28 & 20.4 & \\
\hline $\mathrm{CH}-\mathrm{Gl} 2$ & Core & 9.5 & 125 & 115.5 & \\
\hline DH44 & Core & 5.2 & 125.7 & 120.5 & \\
\hline DH46 & Core & 6.8 & 125.3 & 118.5 & \\
\hline DH48 & Core & 7.5 & 27 & 19.5 & \\
\hline DH50 & Core & 8.1 & 250.5 & 242.4 & \\
\hline DH52 & Core & 7.5 & 50 & 42.5 & \\
\hline DH55 & Core & 6.7 & 125.2 & 118.5 & \\
\hline DH57 & Core & 7.6 & 28 & 20.4 & \\
\hline DH1 & Core & 2.9 & 5.8 & 2.9 & \\
\hline $\mathrm{DH} 2$ & Core & 1.2 & 5.7 & 4.5 & \\
\hline DH3 & Core & 1.6 & 6.2 & 4.6 & \\
\hline DH4 & Core & 2.1 & 6.2 & 4.1 & \\
\hline DH5 & Core & 1.6 & 5.8 & 4.2 & \\
\hline DH6 & Core & 2.3 & 6.7 & 4.4 & \\
\hline DH7 & Core & 1.6 & 5.7 & 4.1 & \\
\hline DH8 & Core & 4 & 9 & 5 & \\
\hline DH9 & Core & 2.5 & 6.5 & 4 & \\
\hline DH10 & Core & 4.2 & 8.2 & 4 & \\
\hline DH11 & Core & 2.8 & 6.4 & 3.6 & \\
\hline DH12 & Core & 2.2 & 6.3 & 4.1 & \\
\hline DH13 & Core & 2.2 & 6 & 3.8 & \\
\hline DH14 & Core & 1.5 & 5.8 & 4.3 & \\
\hline DH15 & Core & 2 & 7.6 & 5.6 & \\
\hline DH16 & Core & 8.6 & 12.6 & 4 & \\
\hline DH17 & Core & 8.5 & 12.5 & 4 & \\
\hline DH18 & Core & 9.2 & 13.3 & 4.1 & \\
\hline
\end{tabular}




\begin{tabular}{|c|c|c|c|c|c|}
\hline Borehole name & Sample type & $\begin{array}{l}\text { Beginning } \\
\text { footage }\end{array}$ & $\begin{array}{l}\text { Ending } \\
\text { footage }\end{array}$ & $\begin{array}{c}\text { Total } \\
\text { footage }\end{array}$ & $\begin{array}{c}\text { Total } \\
\text { depth }(\mathrm{ft})\end{array}$ \\
\hline \multicolumn{6}{|c|}{ Material and Fuels Complex (formerly Argonne National Laboratory West)—Continued } \\
\hline DH19 & Core & 9.5 & 13.3 & 3.8 & \\
\hline DH20 & Core & 10.6 & 14.6 & 4 & \\
\hline DH21 & Core & 2.2 & 6.3 & 4.1 & \\
\hline DH22 & Core & 3.5 & 7.5 & 4 & \\
\hline DH23 & Core & 3.5 & 35 & 31.5 & \\
\hline DH24 & Core & 3.2 & 70 & 66.8 & \\
\hline DH25 & Core & 4.5 & 35 & 30.5 & \\
\hline DH1 & Core & 5 & 19.2 & 14.2 & \\
\hline DH2 & Core & 11.9 & 18.2 & 6.3 & \\
\hline DH5 & Core & 9 & 9.2 & 0.2 & \\
\hline DH6 & Core & 29.2 & 30.9 & 1.7 & \\
\hline ANL-OBS-AQ-014 & Core & 0 & 1,910 & 1,910 & $1,910.00$ \\
\hline EBR-II & Cuttings & 0 & 10 & 10 & \\
\hline USGS 143 & Core & 9 & 829 & 820 & 830.00 \\
\hline \multicolumn{6}{|c|}{ Auxiliary Reactor Area (ARA) } \\
\hline ARA-COR-005 & Core & 9.7 & 855.4 & 845.7 & 860.00 \\
\hline ARA-MON-A-01 & Cuttings & 0 & 630 & 630 & \\
\hline ARA-MON-A-02 & Cuttings & 0 & 620 & 620 & \\
\hline ARA-MON-A-03A & Cuttings & 10 & 650 & 640 & \\
\hline ARA-MON-A-04 & Cuttings & 0 & 485 & 485 & \\
\hline STF-PIE-AQ-01 & Core & 19 & 713.2 & 694.2 & 694.20 \\
\hline STF-PIE-AQ-02 & Core & 10.5 & 549.5 & 539 & 539.00 \\
\hline \multicolumn{6}{|c|}{ Mountain Home Air Base Idaho (about 165 mi southwest of the CFA) } \\
\hline MHTH-1 & Core & 1325 & 4403 & 3078 & $4,403.00$ \\
\hline \multicolumn{6}{|c|}{ Mud Lake Landfill (ML) (about 1 mi northwest of Mud Lake, ID) } \\
\hline ML-1 & Cuttings & 0 & 90 & 90 & \\
\hline \multicolumn{6}{|c|}{ New Production Reactor (NPR) } \\
\hline A-26 & Core $/ 45^{\circ}$-angle hole & 4.5 & 240 & 235.5 & \\
\hline DC-1 & Core & 2.1 & 300 & 297.9 & \\
\hline DC-2 & Core & 3.1 & 296.9 & 293.8 & \\
\hline DC-3 & Core & 1.5 & 300.1 & 298.6 & \\
\hline DC-4 & Core & 3.5 & 300 & 296.5 & \\
\hline F-1 & Core & 4 & 200 & 196 & \\
\hline $\mathrm{F}-2$ & Core & 2.4 & 50 & 47.6 & \\
\hline F-3 & Core & 12.7 & 50 & 37.3 & \\
\hline F-4 & Core & 1.7 & 50 & 48.3 & \\
\hline F-5 & Core & 2 & 50.1 & 48.1 & \\
\hline F-6 & Core & 5.3 & 50.4 & 45.1 & \\
\hline
\end{tabular}




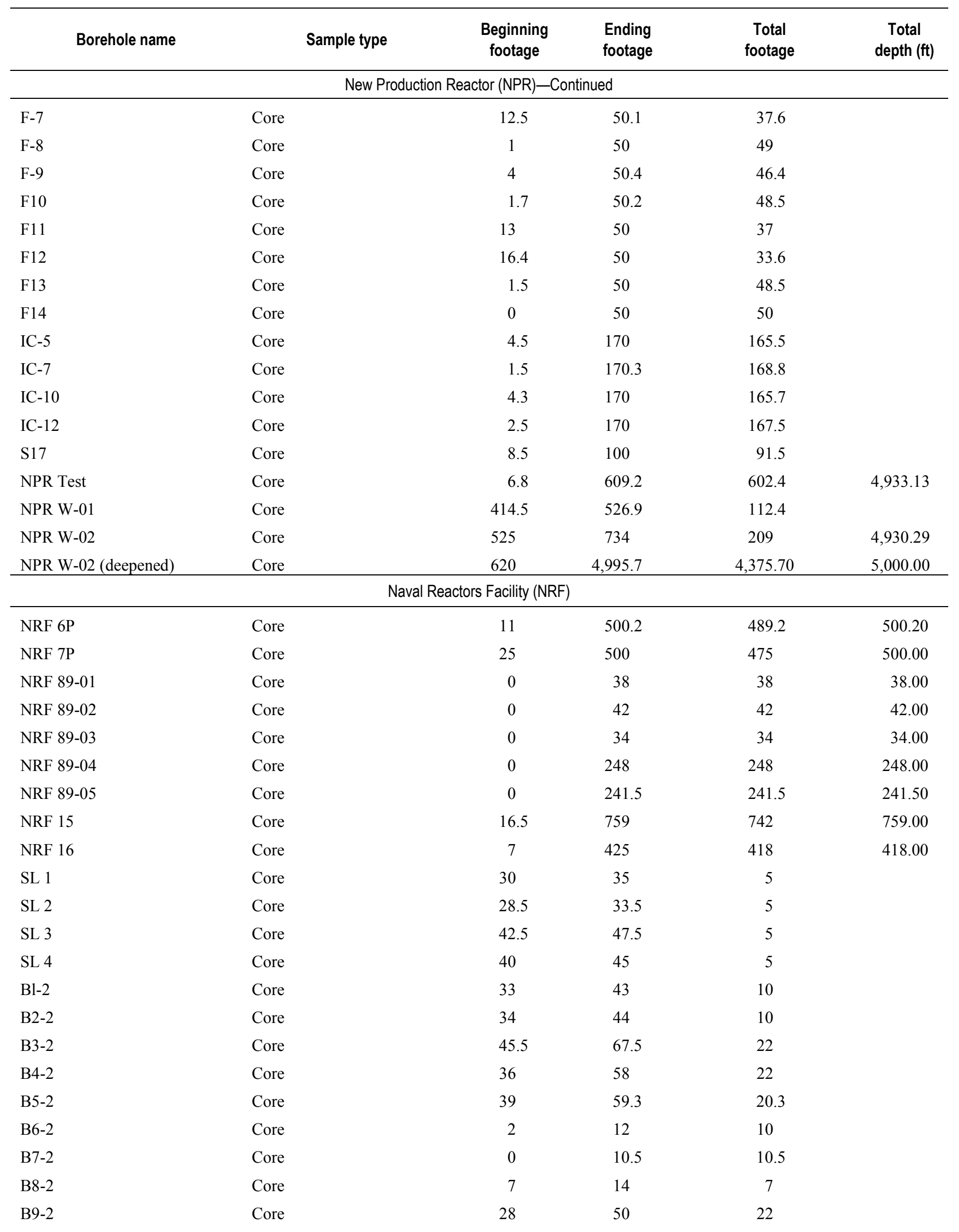




\begin{tabular}{|c|c|c|c|c|c|}
\hline Borehole name & Sample type & $\begin{array}{l}\text { Beginning } \\
\text { footage }\end{array}$ & $\begin{array}{l}\text { Ending } \\
\text { footage }\end{array}$ & $\begin{array}{c}\text { Total } \\
\text { footage }\end{array}$ & $\begin{array}{c}\text { Total } \\
\text { depth (ft) }\end{array}$ \\
\hline \multicolumn{6}{|c|}{ Naval Reactors Facility (NRF)-Continued } \\
\hline $\mathrm{B} 10-2$ & Core & 43 & 65 & 22 & \\
\hline B11-2 & Core & 36 & 58 & 22 & \\
\hline B12-2 & Core & 4 & 9 & 5 & \\
\hline B13-2 & Core & 2 & 12 & 10 & \\
\hline B14-2 & Core & 3 & 13 & 10 & \\
\hline B15-2 & Core & 3 & 13 & 10 & \\
\hline B16-2 & Core & 40 & 50 & 10 & \\
\hline B18-2 & Core & 36.5 & 96.1 & 59.6 & \\
\hline Bl-1 & Core & 32 & 42 & 10 & \\
\hline B2-1 & Core & 23 & 33 & 10 & \\
\hline B3-1 & Core & 23 & 34 & 11 & \\
\hline B4-1 & Core & 24.5 & 47 & 22.5 & \\
\hline B5-1 & Core & 39.5 & 57 & 17.5 & \\
\hline B6-1 & Core & 20.5 & 30.5 & 10 & \\
\hline B7-1 & Core & 26 & 36 & 10 & \\
\hline B8-1 & Core & 34.6 & 56.6 & 22 & \\
\hline B9-1 & Core & 31.5 & 41.5 & 10 & \\
\hline B10-1 & Core & 26 & 38 & 12 & \\
\hline B11-1 & Core & 31 & 52.3 & 21.3 & \\
\hline B12-1 & Core & 21 & 43 & 22 & \\
\hline B13-1 & Core & 21 & 31.5 & 10.5 & \\
\hline B14-1 & Core & 23.5 & 33.5 & 10 & \\
\hline B15-1 & Core & 24.5 & 34.5 & 10 & \\
\hline B16-1 & Core & 34 & 56 & 22 & \\
\hline B17-1 & Core & 50.2 & 59 & 8.8 & \\
\hline B18-1 & Core & 33 & 250.6 & 217.6 & \\
\hline SSG Prod. & Cuttings & 10 & 1,340 & 1,330 & \\
\hline \multicolumn{6}{|c|}{ Idaho National Laboratory--unknown areas } \\
\hline A1 & Cuttings & 0 & 10 & 10 & \\
\hline A2 & Cuttings & 0 & 5 & 5 & \\
\hline A3 & Cuttings & 0 & 3.5 & 3.5 & \\
\hline A4 & Cuttings & 0 & 4 & 4 & \\
\hline $5 \mathrm{~A}$ & Cuttings & 0 & 40 & 40 & \\
\hline $5 \mathrm{~B}$ & Cuttings & 0 & 35 & 35 & \\
\hline $5 \mathrm{C}$ & Cuttings & 0 & 8 & 8 & \\
\hline $5 \mathrm{D}$ & Cuttings & 0 & 58 & 58 & \\
\hline \multicolumn{6}{|c|}{ Power Burst Facility (PBF) } \\
\hline ТН 23 & Core & 24.7 & 41.5 & 16.8 & \\
\hline TH 27 & Core & 5.8 & 58.5 & 52.7 & \\
\hline TH 28 & Core & 6.4 & 69 & 62.6 & \\
\hline
\end{tabular}




\begin{tabular}{|c|c|c|c|c|c|}
\hline Borehole name & Sample type & $\begin{array}{l}\text { Beginning } \\
\text { footage }\end{array}$ & $\begin{array}{l}\text { Ending } \\
\text { footage }\end{array}$ & $\begin{array}{c}\text { Total } \\
\text { footage }\end{array}$ & $\begin{array}{c}\text { Total } \\
\text { depth }(\mathrm{ft})\end{array}$ \\
\hline \multicolumn{6}{|c|}{ Power Burst Facility (PBF)-Continued } \\
\hline TH 32 & Core & 3.8 & 13.6 & 9.8 & \\
\hline TH 44 & Core & 4.3 & 64.7 & 60.4 & \\
\hline TH 92 & Core & 13.6 & 49 & 35.4 & \\
\hline TH 100 & Core & 4.4 & 44.7 & 40.3 & \\
\hline PBF-MON-A-004 & Cuttings & 20 & 595 & 575 & \\
\hline PBF-MON-A-005 & Cuttings & 15 & 545 & 530 & \\
\hline \multicolumn{6}{|c|}{ Radioactive Waste Management Area (RWMC) } \\
\hline Acid Pit-P01 & Sediment & 2 & 16 & 14 & \\
\hline Acid Pit-P02 & Sediment & 2 & 16 & 14 & \\
\hline Acid Pit-P03 & Sediment & 2 & 16 & 14 & \\
\hline Acid Pit-P04 & Sediment & 2 & 16 & 17 & \\
\hline Acid Pit-P05 & Sediment & 2 & 16 & 20 & \\
\hline Acid Pit-P06 & Sediment & 2 & 16 & 16 & \\
\hline B03N21 & Core & 6.3 & 171.1 & 164.8 & \\
\hline B07N21 & Core & 6.8 & 182 & 175.2 & \\
\hline B09GN11 & Core & 19.5 & 179.4 & 159.9 & \\
\hline B10G11 & Core & 11.8 & 178.2 & 166.4 & \\
\hline B10N21 & Core & 69.7 & 174.2 & 104.5 & \\
\hline B14N21 & Core & 5.1 & 176.6 & 171.5 & \\
\hline \multirow[t]{2}{*}{ BG-76-1 } & Core & 84.7 & 125.5 & 40.8 & 228.30 \\
\hline & Processed sediment & 205.9 & 245.8 & 39.9 & \\
\hline \multirow[t]{3}{*}{ BG-76-2 } & Core & 18.1 & 152.6 & 134.5 & 252.50 \\
\hline & Core & 218 & 222 & 4 & \\
\hline & Processed sediment & 223 & 243.9 & 20.9 & \\
\hline \multirow[t]{5}{*}{ BG-76-3 } & Core & 22.2 & 95 & 72.8 & 240.00 \\
\hline & Processed sediment & 99.3 & 119.7 & 20.4 & \\
\hline & Core & 120 & 130.8 & 10.8 & \\
\hline & Core & 216 & 221.8 & 5.8 & \\
\hline & Processed sediment & 223.6 & 240.4 & 16.8 & \\
\hline \multirow[t]{2}{*}{ BG-76-4 } & Core & 11.2 & 114.2 & 103 & 215.00 \\
\hline & Processed sediment & 232.3 & 234.9 & 2.6 & \\
\hline \multirow[t]{3}{*}{ BG-76-4A } & Core & 11.2 & 101.3 & 90.1 & 254.00 \\
\hline & Core & 196.8 & 226 & 29.2 & \\
\hline & Processed sediment & 226 & 243.4 & 17.4 & \\
\hline \multirow[t]{4}{*}{ BG-76-5 } & Core & 10 & 122 & 112 & 245.00 \\
\hline & Processed sediment & 95.9 & 121.2 & 25.3 & \\
\hline & Core & 211.3 & 219.8 & 8.5 & \\
\hline & Processed sediment & 223.7 & 241.2 & 17.5 & \\
\hline BG-76-6 & Core & 9.1 & 243.6 & 234.5 & 243.80 \\
\hline BG-77-1 & Core & 6 & 598 & 592 & 600.00 \\
\hline
\end{tabular}




\begin{tabular}{|c|c|c|c|c|c|}
\hline Borehole name & Sample type & $\begin{array}{l}\text { Beginning } \\
\text { footage }\end{array}$ & $\begin{array}{l}\text { Ending } \\
\text { footage }\end{array}$ & $\begin{array}{c}\text { Total } \\
\text { footage }\end{array}$ & $\begin{array}{c}\text { Total } \\
\text { depth (ft) }\end{array}$ \\
\hline \multicolumn{6}{|c|}{ Radioactive Waste Management Area (RWMC)_Continued } \\
\hline \multirow[t]{2}{*}{ BG-77-2 } & Core & 19.8 & 87 & 67.2 & 87.00 \\
\hline & Core & 193 & 202.2 & 9.2 & 87.40 \\
\hline RWMC-78-1 & Core & 23 & 78 & 55 & 82.00 \\
\hline \multirow[t]{3}{*}{ RWMC-78-2 } & Core & 4.7 & 97 & 92.3 & 252.60 \\
\hline & Core & 106 & 230.1 & 124.1 & \\
\hline & Processed sediment & 230.1 & 243.1 & 13 & \\
\hline \multirow[t]{2}{*}{ RWMC-78-3 } & Core & 5.5 & 224.5 & 219 & 248.00 \\
\hline & Processed sediment & 224.5 & 242.4 & 17.9 & \\
\hline \multirow[t]{4}{*}{ RWMC-78-5 } & Core & 22 & 96.7 & 74.7 & 250.00 \\
\hline & Processed sediment & 99 & 112.4 & 13.4 & \\
\hline & Core & 129.2 & 224.7 & 95.5 & \\
\hline & Processed sediment & 226.5 & 240.3 & 13.8 & \\
\hline \multirow[t]{3}{*}{ RWMC-79-1 } & Core & 4.8 & 129.2 & 124.4 & 244.00 \\
\hline & Processed sediment & 129.2 & 141.7 & 12.5 & \\
\hline & Core & 142.9 & 236.9 & 94 & \\
\hline \multirow[t]{3}{*}{ RWMC-79-2 } & Core & 18.5 & 98.7 & 80.2 & 222.70 \\
\hline & Processed sediment & 99.9 & 103 & 3.1 & \\
\hline & Core & 106.5 & 222.9 & 116.4 & \\
\hline \multirow[t]{2}{*}{ RWMC-79-3 } & Core & 17.2 & 169.1 & 151.9 & 262.00 \\
\hline & Processed sediment & 235 & 251.7 & 16.7 & \\
\hline \multirow[t]{3}{*}{ USGS 91} & Core & 8 & 111 & 103 & 255.00 \\
\hline & Core & 131.8 & 234 & 102.2 & \\
\hline & Core & 245 & 254 & 9 & \\
\hline \multirow[t]{2}{*}{ USGS 92} & Core & 19.3 & 226.4 & 207.1 & 247.00 \\
\hline & Processed sediment & 220.5 & 249.7 & 29.2 & \\
\hline \multirow[t]{2}{*}{ USGS 93} & Processed sediment & 0 & 12.1 & 12.1 & \\
\hline & Core & 14 & 246.1 & 232.1 & 246.25 \\
\hline \multirow[t]{4}{*}{ USGS 93A } & Core & 25.8 & 100.5 & 74.7 & \\
\hline & Processed sediment & 100.5 & 109.1 & 8.6 & \\
\hline & Core & 109.5 & 221.7 & 112.2 & \\
\hline & Processed sediment & 227.9 & 233.2 & 5.3 & \\
\hline \multirow[t]{4}{*}{ USGS 94} & Core & 12.4 & 96.3 & 83.9 & \\
\hline & Core & 112.6 & 223.3 & 110.7 & \\
\hline & Processed sediment & 231.3 & 244.8 & 13.5 & \\
\hline & Core & 247 & 302.3 & 55.3 & \\
\hline \multirow[t]{2}{*}{ USGS 95} & Processed sediment & 0 & 24 & 24 & \\
\hline & Core & 24 & 243 & 219 & \\
\hline USGS 96 & Core & 15 & 236.3 & 221.3 & \\
\hline \multirow[t]{2}{*}{ USGS 96A } & Core & 22.3 & 67.9 & 45.6 & \\
\hline & Core & 87.5 & 121 & 33.5 & \\
\hline
\end{tabular}




\begin{tabular}{|c|c|c|c|c|c|}
\hline Borehole name & Sample type & $\begin{array}{l}\text { Beginning } \\
\text { footage }\end{array}$ & $\begin{array}{l}\text { Ending } \\
\text { footage }\end{array}$ & $\begin{array}{c}\text { Total } \\
\text { footage }\end{array}$ & $\begin{array}{c}\text { Total } \\
\text { depth (ft) }\end{array}$ \\
\hline \multicolumn{6}{|c|}{ Radioactive Waste Management Area (RWMC)_Continued } \\
\hline \multirow[t]{4}{*}{ USGS 96B } & Core & 20 & 105.4 & 84.7 & \\
\hline & Processed sediment & 106.3 & 124.9 & 18.6 & \\
\hline & Core & 128.8 & 214.5 & 85.7 & \\
\hline & Processed sediment & 221 & 227.4 & 6.4 & \\
\hline USGS 104 & Cuttings & 15 & 201 & 186 & \\
\hline USGS 118 & Core & 0 & 569.9 & 569.9 & 569.90 \\
\hline \multirow[t]{2}{*}{ RWMC-88-1 D } & Core & 28.6 & 210.9 & 181.4 & \\
\hline & Core & 220.3 & 230.1 & 9.8 & \\
\hline RWMC-88-020 (8802D) & Core & 6 & 206.4 & 200.4 & \\
\hline \multirow[t]{2}{*}{ RWMC-89-01 D } & Core & 31.6 & 40.9 & 9.3 & \\
\hline & & 88.8 & 113 & 24.2 & \\
\hline PIT-9-P02 & Sediment & 16 & 14 & & \\
\hline PIT-9-P03 & Sediment & 8 & 6 & & \\
\hline PIT-9-P04 & Sediment & 16 & 14 & & \\
\hline PIT-9-P05 & Sediment & 20 & 18 & & \\
\hline PIT-9-P06 & Sediment & 4 & 2 & & \\
\hline PIT-9-P07 & Sediment & 14 & 12 & & \\
\hline PIT-9-P08 & Sediment & 14 & 12 & & \\
\hline SIP W-05 & Core & 224 & 232 & 8.0 & \\
\hline SIP W-06 & Core & 233 & 247 & 14.0 & \\
\hline SIP W-09 & Core & 284 & 291 & 7.0 & \\
\hline SIP W-11 & Core & 200 & 208 & 8.0 & \\
\hline SIP W-13 & Core & 258 & 267 & 9.0 & \\
\hline SIP W-17 \&17A & Core & 269 & 283 & 14.0 & \\
\hline SIP W-18 & Core & 248 & 257 & 9.0 & \\
\hline D-10 & Core & 96.5 & 221.5 & 125 & \\
\hline D-15 & \multicolumn{4}{|c|}{ Core-miscellaneous pieces } & \\
\hline $\mathrm{C}-1(\mathrm{Cl})$ & Core & 9.5 & 663.5 & 654 & \\
\hline C-1A (CIA, Cl-A) & Core & 7.2 & 1805 & $1,797.80$ & $1,805.00$ \\
\hline P-02 & Cuttings & 111.4 & 175 & 63.6 & \\
\hline WWW\#1 (WWWI, VZ6A) & Core & 9.9 & 265 & 255.1 & \\
\hline WWW\#2 (WWW2, VZ6) & Core & 10.5 & 109.7 & 99.2 & \\
\hline USGS 129 & core & 9.2 & 779 & 770 & 779.00 \\
\hline USGS 132 & core & 9 & 1,238 & 1,229 & $1,238.00$ \\
\hline \multicolumn{6}{|c|}{ Sugar City Exploration Well } \\
\hline Corehole 3 & Core & 0 & 2,283 & 2,283 & \\
\hline \multicolumn{6}{|c|}{ Test Area North (Test Area North) } \\
\hline TCH \#1 & Core & 46 & 600 & 554 & 600.00 \\
\hline TCH \#2 Piezo A & Core & 47.4 & $1,113.5$ & $1,066.1$ & $1,113.50$ \\
\hline
\end{tabular}




\begin{tabular}{|c|c|c|c|c|c|}
\hline Borehole name & Sample type & $\begin{array}{l}\text { Beginning } \\
\text { footage }\end{array}$ & $\begin{array}{l}\text { Ending } \\
\text { footage }\end{array}$ & $\begin{array}{c}\text { Total } \\
\text { footage }\end{array}$ & $\begin{array}{c}\text { Total } \\
\text { depth (ft) }\end{array}$ \\
\hline \multicolumn{6}{|c|}{ Test Area North (Test Area North)-Continued } \\
\hline GIN \#1 & Cuttings & 10 & 372 & 362 & \\
\hline GIN \#2 & Cuttings & 10 & 402 & 392 & \\
\hline GIN \#3 & Cuttings & 10 & 375 & 365 & \\
\hline GIN \#4 & Cuttings & 10 & 306 & 296 & \\
\hline GIN \#5 & Core & 30.5 & 430.2 & 399.7 & 430.00 \\
\hline GIN \#6 & Core & 59 & 200.1 & 141.1 & 200.10 \\
\hline TAN \#27 & Cuttings & 45 & 205 & 160 & \\
\hline TAN \#27(con't) & Core & 205.5 & 249.5 & 44 & 253.70 \\
\hline TAN \#28 & Cuttings & 0 & 255 & 255 & \\
\hline TAN \#29 & Cuttings & 0 & 265 & 265 & \\
\hline TAN \#30 & Cuttings & 0 & 170 & 170 & \\
\hline TAN \#30A & Cuttings & 30 & 320 & 290 & \\
\hline MW-2 & Cuttings & 0 & 250 & 250 & \\
\hline USGS 126A & Core & 5.6 & 519.5 & 513.9 & 648 \\
\hline Corehole-2A & Core & 40 & 3,000 & 2,960 & $3,000.00$ \\
\hline $\mathrm{A} 1$ & Core & 42.9 & 57.9 & 15 & \\
\hline A2 & Core & 45.3 & 60.3 & 15 & \\
\hline A3 & Core & 40.8 & 50.8 & 10 & \\
\hline A4 & Core & 48 & 58 & 10 & \\
\hline A6 & Core & 44.2 & 54.2 & 10 & \\
\hline A7 & Core & 45 & 62 & 17 & \\
\hline A9 & Core & 45.2 & 60.2 & 15 & \\
\hline A10 & Core & 48.7 & 60.7 & 12 & \\
\hline A11 & Core & 42 & 52 & 10 & \\
\hline A12 & Core & 41.5 & 51.5 & 10 & \\
\hline A13 & Core & 44.6 & 54.6 & 10 & \\
\hline A14 & Core & 46.1 & 56.1 & 10 & \\
\hline A15 & Core & 48 & 58 & 10 & \\
\hline A16 & Core & 45.8 & 63.8 & 18 & \\
\hline A17 & Core & 43.8 & 53.8 & 10 & \\
\hline A18 & Core & 40.1 & 50.1 & 10 & \\
\hline $\mathrm{A} 20$ & Core & 45.1 & 55.1 & 10 & \\
\hline $\mathrm{A} 21$ & Core & 41.8 & 51.8 & 10 & \\
\hline A 22 & Core & 40.5 & 50.5 & 10 & \\
\hline A24 & Core & 47.1 & 57.1 & 10 & \\
\hline A25 & Core & 41.1 & 51.1 & 10 & \\
\hline A26 & Core & 44.2 & 59.2 & 15 & \\
\hline $\mathrm{A} 27$ & Core & 42.6 & 52.6 & 10 & \\
\hline A28 & Core & 42.1 & 52.1 & 10 & \\
\hline A31 & Core & 39.8 & 49.8 & 10 & \\
\hline
\end{tabular}




\begin{tabular}{|c|c|c|c|c|c|}
\hline Borehole name & & $\begin{array}{l}\text { Beginning } \\
\text { footage }\end{array}$ & $\begin{array}{l}\text { Ending } \\
\text { footage }\end{array}$ & $\begin{array}{c}\text { Total } \\
\text { footage }\end{array}$ & $\begin{array}{c}\text { Total } \\
\text { depth (ft) }\end{array}$ \\
\hline \multicolumn{6}{|c|}{ Test Area North (Test Area North)-Continued } \\
\hline A 32 & Core & 39.9 & 49.9 & 10 & \\
\hline A33 & Core & 40.3 & 50.3 & 10 & \\
\hline A34 & Core & 39.5 & 49.5 & 10 & \\
\hline A35 & Core & 41.7 & 51.7 & 10 & \\
\hline A36 & Core & 43.3 & 53.3 & 10 & \\
\hline A 37 & Core & 41.1 & 51.1 & 10 & \\
\hline A38 & Core & 45 & 55 & 10 & \\
\hline A39 & Core & 43.7 & 53.7 & 10 & \\
\hline $\mathrm{C} 1$ & Core & 49 & 64 & 15 & \\
\hline $\mathrm{C} 5$ & Core & 42 & 52 & 10 & \\
\hline $\mathrm{C} 7$ & Core & 46 & 56 & 10 & \\
\hline $\mathrm{C} 12$ & Core & 44.2 & 54.2 & 10 & \\
\hline $\mathrm{C} 14$ & Core & 41.5 & 49.5 & 8 & \\
\hline $\mathrm{C} 15$ & Core & 42.5 & 57.5 & 15 & \\
\hline $\mathrm{C} 22$ & Core & 42.5 & 52.5 & 10 & \\
\hline $\mathrm{C} 23$ & Core & 42 & 51 & 9 & \\
\hline $\mathrm{C} 24$ & Core & 47.5 & 63.5 & 16 & \\
\hline $\mathrm{C} 31$ & Core & 41.5 & 65.5 & 24 & \\
\hline $\mathrm{C} 32$ & Core & 41.5 & 61.5 & 20 & \\
\hline $\mathrm{C} 33$ & Core & 45.3 & 55.3 & 10 & \\
\hline $\mathrm{C} 34$ & Core & 46.4 & 63.4 & 17 & \\
\hline $\mathrm{C} 35$ & Core & 47.1 & 57.1 & 10 & \\
\hline $\mathrm{C} 36$ & Core & 42.2 & 52.2 & 10 & \\
\hline $\mathrm{C} 37$ & Core & 47.1 & 57.1 & 10 & \\
\hline C38 & Core & 42.6 & 56.6 & 14 & \\
\hline C39 & Core & 41.7 & 51.7 & 10 & \\
\hline $\mathrm{C} 40$ & Core & 44.2 & 64.2 & 20 & \\
\hline C41 & Core & 42.2 & 52.2 & 10 & \\
\hline $\mathrm{C} 42$ & Core & 42 & 52 & 10 & \\
\hline $\mathrm{C} 43$ & Core & 41.6 & 51.6 & 10 & \\
\hline $\mathrm{C} 44$ & Core & 41.7 & 51.7 & 10 & \\
\hline $\mathrm{C} 45$ & Core & 41.2 & 51.2 & 10 & \\
\hline $\mathrm{C} 46$ & Core & 41.7 & 51.7 & 10 & \\
\hline $\mathrm{C} 47$ & Core & 41.1 & 51.1 & 10 & \\
\hline $\mathrm{C} 48$ & Core & 41.1 & 51.1 & 10 & \\
\hline C49 & Core & 41 & 51 & 10 & \\
\hline C50 & Core & 41.3 & 51.3 & 10 & \\
\hline C51 & Core & 40.8 & 50.8 & 10 & \\
\hline D1 & Core & 30.2 & 40.2 & 10 & \\
\hline D4 & Core & 38 & 58 & 20 & \\
\hline
\end{tabular}




\begin{tabular}{|c|c|c|c|c|c|}
\hline Borehole name & & $\begin{array}{l}\text { Beginning } \\
\text { footage }\end{array}$ & $\begin{array}{l}\text { Ending } \\
\text { footage }\end{array}$ & $\begin{array}{c}\text { Total } \\
\text { footage }\end{array}$ & $\begin{array}{c}\text { Total } \\
\text { depth (ft) }\end{array}$ \\
\hline \multicolumn{6}{|c|}{ Test Area North (Test Area North)_Continued } \\
\hline D5 & Core & 29 & 39 & 10 & \\
\hline D6 & Core & 32.5 & 42.5 & 10 & \\
\hline D8 & Core & 47 & 57 & 10 & \\
\hline D9 & Core & 29 & 39 & 10 & \\
\hline D10 & Core & 30.5 & 40.5 & 10 & \\
\hline D12 & Core & 44.5 & 54.5 & 10 & \\
\hline D13 & Core & 28 & 38 & 10 & \\
\hline D14 & Core & 30.7 & 40.7 & 10 & \\
\hline D16 & Core & 45.5 & 60.5 & 15 & \\
\hline D17 & Core & 35.4 & 45.4 & 10 & \\
\hline D18 & Core & 46.6 & 56.6 & 10 & \\
\hline D20 & Core & 37.6 & 47.6 & 10 & \\
\hline D22 & Core & 43.8 & 58.8 & 15 & \\
\hline D23 & Core & 37.6 & 47.6 & 10 & \\
\hline D26 & Core & 33.5 & 43.5 & 10 & \\
\hline D29 & Core & 31.9 & 41.9 & 10 & \\
\hline D30 & Core & 38 & 54 & 16 & \\
\hline D32 & Core & 32 & 42 & 10 & \\
\hline D33 & Core & 38 & 48 & 10 & \\
\hline D35 & Core & 27.8 & 37.8 & 10 & \\
\hline D36 & Core & 40.2 & 50.2 & 10 & \\
\hline D37 & Core & 57.3 & 67.3 & 10 & \\
\hline D39 & Core & 36.9 & 46.9 & 10 & \\
\hline D40 & Core & 45.7 & 55.7 & 10 & \\
\hline D42 & Core & 28 & 38 & 10 & \\
\hline D43 & Core & 29 & 39 & 10 & \\
\hline D44 & Core & 41.6 & 51.6 & 10 & \\
\hline D45 & Core & 32.3 & 42.3 & 10 & \\
\hline D46 & Core & 34.9 & 44.9 & 10 & \\
\hline D47 & Core & 45.6 & 65.6 & 20 & \\
\hline D48 & Core & 30.2 & 40.2 & 10 & \\
\hline D49 & Core & 33.6 & 43.6 & 10 & \\
\hline D50 & Core & 46.5 & 67.5 & 21 & \\
\hline D51 & Core & 28.2 & 38.2 & 10 & \\
\hline D52 & Core & 43.3 & 68.3 & 25 & \\
\hline D53 & Core & 30.8 & 40.8 & 10 & \\
\hline D54 & Core & 30 & 40 & 10 & \\
\hline D55 & Core & 28 & 38 & 10 & \\
\hline D57 & Core & 37.7 & 47.7 & 10 & \\
\hline E1 & Core & 42.1 & 52.1 & 10 & \\
\hline
\end{tabular}




\begin{tabular}{|c|c|c|c|c|c|}
\hline Borehole name & Sample type & $\begin{array}{l}\text { Beginning } \\
\text { footage }\end{array}$ & $\begin{array}{l}\text { Ending } \\
\text { footage }\end{array}$ & $\begin{array}{c}\text { Total } \\
\text { footage }\end{array}$ & $\begin{array}{c}\text { Total } \\
\text { depth (ft) }\end{array}$ \\
\hline \multicolumn{6}{|c|}{ Test Area North (Test Area North)-Continued } \\
\hline E2 & Core & 40.9 & 50.9 & 10 & \\
\hline E3 & Core & 41.2 & 51.2 & 10 & \\
\hline E4 & Core & 41.1 & 51.1 & 10 & \\
\hline E5 & Core & 41.3 & 51.4 & 10.1 & \\
\hline E6 & Core & 41.7 & 51.7 & 10 & \\
\hline E9 & Core & 41.1 & 61.1 & 20 & \\
\hline E10 & Core & 41.2 & 51.2 & 10 & \\
\hline E11 & Core & 41.6 & 51.6 & 10 & \\
\hline E13 & Core & 41.1 & 51.1 & 10 & \\
\hline E14 & Core & 41.7 & 51.7 & 10 & \\
\hline TAN 2271 & Core & 33 & 289 & 250 & 289.00 \\
\hline \multicolumn{6}{|c|}{ Wendell-RASA Test Hole } \\
\hline Wendell-RASA Test Hole & Core & & 6 & 1093 & $1,087.00$ \\
\hline \multicolumn{6}{|c|}{ Corehole 1} \\
\hline Corehole 1, Exploration Well 1 & Core & & 40 & 2000 & $1,960.00$ \\
\hline \multicolumn{6}{|c|}{ Miscellaneous } \\
\hline CLC-3 & Sediment & & 0 & 19 & 19.00 \\
\hline CLC-4B & Sediment & & 0 & 12 & 12.00 \\
\hline CLC-5 & Sediment & & 0 & 11.8 & 11.80 \\
\hline CLC-7 & Sediment & & 0 & 7.5 & 7.50 \\
\hline CLC-8TR & Sediment & & 0 & 18 & 18.00 \\
\hline CLC-9 & Sediment & & 0 & 16 & 16.00 \\
\hline CLC-10 & Sediment & & 0 & 14 & 14.00 \\
\hline \multicolumn{6}{|c|}{ Idaho National Laboratory not associated with facility } \\
\hline USGS 134 & Core & 10.5 & 949 & & 938.50 \\
\hline USGS 138 & Core & 0 & 334 & 333.6 & 334.00 \\
\hline USGS 139 & Core & 6 & 788 & 782 & 788.0 \\
\hline USGS 142 & Core & 5 & 1,880 & 1,875 & $1,880.00$ \\
\hline Middle 2051 & Core & 127 & 1,179 & 1,053 & $1,179.00$ \\
\hline
\end{tabular}


Table 3. Summary of investigations on geology, paleomagnetism, and stratigraphy of the eastern Snake River Plain and Idaho National Laboratory, Idaho.

[Reference: Many listed publications are available at https://pubs.er.usgs.gov/. Abbreviations: INL, Idaho National Laboratory; ESRP, eastern Snake River Plain; INTEC, Idaho Nuclear Technology and Engineering Center (also known as Idaho Chemical Processing Plant [ICPP]); CFA, Central Facilities Area; RWMC, Radioactive Waste Management Complex; TAN, Test Area North; NPR, New Production Reactor; NRF, Naval Reactors Facility; SRP, Snake River Plain; USGS, U.S. Geological Survey]

\begin{tabular}{|c|c|c|}
\hline Reference & Core analyzed & Types of analyses \\
\hline $\begin{array}{l}\text { Barraclough and } \\
\text { others, } 1976\end{array}$ & BG- $88,89,90,91,92,93,94,95,96$ & $\begin{array}{l}\text { Mineralogy and grainsize distribution of } \\
\text { sedimentary interbeds }\end{array}$ \\
\hline $\begin{array}{l}\text { Bartholomay and } \\
\text { others, } 1989\end{array}$ & UZ6A; PW-1, 2, 3, 4, 6, 7, and 8 & $\begin{array}{l}\text { Mineralogy and grainsize distribution of } \\
\text { sedimentary interbeds }\end{array}$ \\
\hline $\begin{array}{l}\text { Bartholomay } \\
1990 \mathrm{~b}\end{array}$ & TAN CH-1 & Mineralogy of sedimentary interbed material \\
\hline $\begin{array}{l}\text { Bestland and } \\
\text { others, } 2002\end{array}$ & Corehole 2-2A & $\begin{array}{l}\text { Sedimentary interbeds in corehole } 2-2 \mathrm{~A} \text { in the Big } \\
\text { Lost River trough. Carbon and oxygen isotopes } \\
\text { of clay material. }\end{array}$ \\
\hline Blair, 2002 & $\begin{array}{l}\text { Corehole 2-2A, WO-2, C-1A, ANL- } \\
\text { OBS--A-001 }\end{array}$ & $\begin{array}{l}\text { Grain-size of sedimentary interbeds, zircon } \\
\text { analyses. }\end{array}$ \\
\hline $\begin{array}{c}\text { Champion and } \\
\text { others, } 1981\end{array}$ & NPR Test & $\begin{array}{l}\text { Radiometric ages and paleomagnetism at corehole } \\
\text { Site E (NPR Test) }\end{array}$ \\
\hline $\begin{array}{r}\text { Champion and } \\
\text { others, } 1988\end{array}$ & NPR Test & $\begin{array}{l}\text { Radiometric ages and paleomagnetism at corehole } \\
\text { Site E (NPR Test), description of Big Lost } \\
\text { cryptochron }\end{array}$ \\
\hline $\begin{array}{l}\text { Champion and } \\
\text { Herman, } 2003\end{array}$ & ICPP $213,214,215 ;$ and USGS 128 & $\begin{array}{l}\text { Paleomagnetism of basalt from drill cores at } \\
\text { Vadose zone research park near INTEC }\end{array}$ \\
\hline $\begin{array}{r}\text { Champion and } \\
\text { others, } 2002\end{array}$ & $\begin{array}{l}\text { ANL-OBS-AQ-014; ARA-COR-005; } \\
\text { BG-77-1; C-1A; Corehole 1, 2-2A; } \\
\text { ICPP-COR-A-023; NPR Test/WO- } \\
\text { 2; NRF-6P, 7P, B18-1, 89-04, 89- } \\
\text { 05; TAN CH-1, 2; TRA-05; USGS } \\
\text { 80, 118, 121, 123 }\end{array}$ & $\begin{array}{l}\text { Age of basalt flows from cores throughout the } \\
\text { INL }\end{array}$ \\
\hline $\begin{array}{r}\text { Champion and } \\
\text { others, } 2011\end{array}$ & $\begin{array}{l}\text { ANL-DH-50; ANL-OBS-A-001; } \\
\text { ARA-COR-005; BG-76, 77-1, 93A, } \\
\text { 94A; C1A; Corehole 1, 2-2A; GIN- } \\
\text { 5, 6; ICPP-023, 1795, 1796, 1797, } \\
\text { 1798, 213, 214, 215; Middle 1823, } \\
\text { 2050A, 2051; NPR Test-WO-2; } \\
\text { NRF-B18-1, 6P, 7P, 89-04, 89-05; } \\
\text { PW-13; STF-AQ-01; TAN CH-1, } ; \\
\text { TRA-5, 6; USGS 80, 81,118, 121, } \\
\text { 123, 126A, 127, 128, 129, 130, 131, } \\
\text { 121, 133, 134, 135; VZ6A-WWW-1 }\end{array}$ & $\begin{array}{l}\text { Stratigraphic correlation of southern part of INL } \\
\text { along with paleomagnetic information for many } \\
\text { cores throughout the INL }\end{array}$ \\
\hline $\begin{array}{r}\text { Champion and } \\
\text { others, } 2013\end{array}$ & $\begin{array}{l}\text { NRF B18-1, 89-04, 89-05, 6P, 7P, 15, } \\
\text { 16, and USGS } 133\end{array}$ & $\begin{array}{l}\text { Stratigraphic correlation of wells at NRF and to } \\
\text { wells south of NRF; includes paleomagnetic } \\
\text { data and argon age dates for several cores. }\end{array}$ \\
\hline Doherty, 1979a & Corehole 2-2A & Drilling data, lithologic and geophysical logs \\
\hline Doherty, 1979b & Corehole \#1 & Drilling data, lithologic and geophysical logs \\
\hline
\end{tabular}




\begin{tabular}{|c|c|c|}
\hline Reference & Core analyzed & Types of analyses \\
\hline $\begin{array}{l}\text { Doherty and } \\
\text { others, } 1979 \\
\end{array}$ & $\begin{array}{l}\text { Exploratory geothermal test well } \\
\text { (INEL-1) }\end{array}$ & Drilling data, geophysical, and lithologic logs \\
\hline $\begin{array}{l}\text { Geist and others, } \\
2002\end{array}$ & TAN 33, 34; GIN-5, 6; & $\begin{array}{l}\text { Gechemistry and paleomagnetic inclination data } \\
\text { collected and compared to Lanphere study. }\end{array}$ \\
\hline $\begin{array}{l}\text { Geslin and others, } \\
2002\end{array}$ & $\begin{array}{l}\text { Corehole 2-2A; TAN CH 1, 2; GIN-5, } \\
\text { 6; TAN-34; Borehole 14A }\end{array}$ & $\begin{array}{l}\text { Point counts, detrital zircon analyses, and } \\
\text { mineralogy of sediment from cores in the Big } \\
\text { Lost Trough }\end{array}$ \\
\hline $\begin{array}{l}\text { Gianniny and } \\
\text { others, } 2002\end{array}$ & $\begin{array}{l}\text { Jefferson landfill cores CB 20, 21, 23; } \\
\text { Corehole 2-2A }\end{array}$ & Ostracod identification in sediments cores \\
\hline $\begin{array}{l}\text { Grimm-Chadwick, } \\
2004\end{array}$ & USGS $127,128,129$ & $\begin{array}{l}\text { Stratigraphy, geochemistry, and descriptions of } \\
\text { high K2O flow in cores }\end{array}$ \\
\hline $\begin{array}{l}\text { Hodges and others, } \\
2012\end{array}$ & $\begin{array}{l}\text { USGS } 103,105,108,131,135 \text {; NRF- } \\
\quad 15,16\end{array}$ & $\begin{array}{l}\text { Construction diagrams, lithologic descriptions, } \\
\text { and geophysical logs for coreholes }\end{array}$ \\
\hline $\begin{array}{l}\text { Hodges and others, } \\
2015\end{array}$ & $\begin{array}{l}\text { ARA-COR-005; BG-77-1; NRF-6P, } \\
\text { 16; USGS 128, 131, } 135\end{array}$ & $\begin{array}{l}\text { Age dates for selected flows from selected } \\
\text { coreholes around the INL }\end{array}$ \\
\hline $\begin{array}{l}\text { Hodges and } \\
\quad \text { Champion, } 2016\end{array}$ & USGS $103,105,108,131,136,137 \mathrm{~A}$ & $\begin{array}{l}\text { Stratigraphic correlation of southern part of INL } \\
\text { including paleomagnetic data for several } \\
\text { coreholes. }\end{array}$ \\
\hline $\begin{array}{l}\text { Hughes and others, } \\
2002\end{array}$ & $\begin{array}{l}\text { TAN CH-1, 2; TAN 34; GIN-5, 6; } \\
\text { Corehole 2-2A, USGS 80, 118, } \\
\text { 126A; ML-1; CB-21, 23; ICPP-023; } \\
\text { TRA-05; BG-77-1; C-1A; STF- } \\
\text { PIE-AQ-01; Corehole 1; Argonne-1, } \\
\text { WO-2 }\end{array}$ & Major and trace element analyses of basalt cores \\
\hline $\begin{array}{l}\text { Kuntz and others, } \\
1980\end{array}$ & USGS 94, BG-77-1, 76-6 & $\begin{array}{l}\text { Radiometric dating, paleomagnetism on cores } \\
\text { from RWMC }\end{array}$ \\
\hline $\begin{array}{l}\text { Lanphere and } \\
\text { others, } 1994\end{array}$ & TAN CH 1,2 & $\begin{array}{l}\text { Petrography, age and paleomagnetism of basalt } \\
\text { flows at and near TAN }\end{array}$ \\
\hline $\begin{array}{l}\text { Lanphere and } \\
\text { others, } 1993\end{array}$ & $\begin{array}{l}\text { ICPP-123; NRF 89-04, 89-05; USGS } \\
80\end{array}$ & $\begin{array}{l}\text { Petrography, age and paleomagnetism of basalt } \\
\text { flows at and near NRF }\end{array}$ \\
\hline Mazurek, 2004 & Middle 1823, WO-2 & $\begin{array}{l}\text { Core logs, clay mineralogy of sediments, } \\
\text { geochemistry of basalt and sedimentary } \\
\text { interbeds }\end{array}$ \\
\hline Miller, 2007 & USGS 132 & $\begin{array}{l}\text { Geochemistry, and descriptions of the B flow, and } \\
\text { stratigraphy of corehole USGS } 132\end{array}$ \\
\hline $\begin{array}{l}\text { Morse and } \\
\text { McCurry, } 2002\end{array}$ & C1A, Corehole 2-2A, WO-2, ANL-1 & $\begin{array}{l}\text { Base of the aquifer, alteration in basalts, basalt } \\
\text { geochemical data }\end{array}$ \\
\hline Mudge, 2016 & NRF-15, USGS 142 & $\begin{array}{l}\text { Sedimentary interbed grain size and provinence } \\
\text { and correlation. }\end{array}$ \\
\hline Perkins, 2003 & ICPP-215 & $\begin{array}{l}\text { Hydraulic properties of sedimentary interbeds } \\
\text { near INTEC }\end{array}$ \\
\hline $\begin{array}{l}\text { Perkins and } \\
\text { Nimmo, } 2000\end{array}$ & UZ98-2 & $\begin{array}{l}\text { Hydraulic properties of sedimentary interbeds } \\
\text { near RWMC }\end{array}$ \\
\hline Potter, 2010 & USGS 135 & $\begin{array}{l}\text { Geochemistry, photos, and petrological analyses } \\
\text { of core USGS } 135\end{array}$ \\
\hline
\end{tabular}




\begin{tabular}{|c|c|c|}
\hline Reference & Core analyzed & Types of analyses \\
\hline $\begin{array}{l}\text { Reed and } \\
\text { Bartholomay, } \\
1994\end{array}$ & $\begin{array}{l}\text { CLC-4A; DC-1, 4; GIN-6; HWY-1; } \\
\text { NPR Test; NRF 6P, 7P; PW-7, PW- } \\
\text { 11; RWMC C1A; TAN CH 1, 2; } \\
\text { TRA-5, 8; USGS 30, 80, 81, 99; } \\
\text { 103, 104, 118, 121, 123; WO-2; }\end{array}$ & $\begin{array}{l}22 \text { cores sampled for mineralogy of sedimentary } \\
\text { interbeds }\end{array}$ \\
\hline $\begin{array}{l}\text { Reed and others, } \\
1997\end{array}$ & ICPP 121, ICPP 123 & $\begin{array}{l}\text { Whole rock geochemistry of cores from wells } 121 \\
\text { and } 123 \text {. }\end{array}$ \\
\hline Rightmire, 1984 & BG-93A, 96B & $\begin{array}{l}\text { Mineralogy and grain size of sedimentary } \\
\text { interbeds }\end{array}$ \\
\hline $\begin{array}{r}\text { Rightmire and } \\
\text { Lewis, } 1987\end{array}$ & $\begin{array}{l}\text { BG-76-1, 76-2, 76-3, 76-4, 76-5, 76-6, } \\
\quad 77-1,77-2\end{array}$ & $\begin{array}{l}\text { Unsaturated zone geology, geochemistry of } \\
\text { sediment and alteration products, mineralogy } \\
\text { and grain size of sedimentary interbeds, core } \\
\text { descriptions. }\end{array}$ \\
\hline Scarberry, 2003 & $\begin{array}{l}\text { ARA-COR-005; BG-77-1; C1A; NPR } \\
\text { Test; STF-AQ-01; USGS } 118\end{array}$ & $\begin{array}{l}\text { Geochemistry of the F flow (now referred to as } \\
\text { the Big Lost Reversed Polarity Cryptochron } \\
\text { flows) and distribution in several coreholes at } \\
\text { the INL }\end{array}$ \\
\hline $\begin{array}{l}\text { Shervais and } \\
\text { others, } 2006\end{array}$ & INL, TAN cores & $\begin{array}{l}\text { Cyclic geochemical variations in basalt in TAN } \\
\text { drill cores }\end{array}$ \\
\hline $\begin{array}{l}\text { Twining and } \\
\text { others, } 2008\end{array}$ & $\begin{array}{l}\text { USGS 126a, 126b, 127, 128, 129, 130, } \\
\quad 131,132,133 \text { and } 134\end{array}$ & $\begin{array}{l}\text { Construction diagrams, lithological, and } \\
\text { geophysical logs for coreholes }\end{array}$ \\
\hline $\begin{array}{l}\text { Twining and } \\
\text { others, } 2012\end{array}$ & USGS 136 & $\begin{array}{l}\text { Core log, photos and geophysical logs for well } \\
\text { USGS } 136\end{array}$ \\
\hline $\begin{array}{l}\text { Twining and } \\
\text { others, } 2014\end{array}$ & USGS 140 & $\begin{array}{l}\text { Core log, photos and geophysical logs for well } \\
\text { USGS } 140\end{array}$ \\
\hline $\begin{array}{l}\text { Twining and } \\
\text { others, } 2016\end{array}$ & TAN 2271, 2272 & $\begin{array}{l}\text { Core log, photos and geophysical logs for well } \\
\text { TAN } 2271 \text { and } 2272\end{array}$ \\
\hline $\begin{array}{l}\text { Twining and } \\
\text { others, } 2017\end{array}$ & USGS 142 & $\begin{array}{l}\text { Core log, photos and geophysical logs for well } \\
\text { USGS } 142\end{array}$ \\
\hline Wetmore, 1998 & $\begin{array}{l}\text { BG-77-1, C1A, STF-PIE-AQ-1, } \\
\text { USGS } 118\end{array}$ & $\begin{array}{l}\text { Geochemistry of basalt flows to determine } \\
\text { distribution in several coreholes at the INL }\end{array}$ \\
\hline Winfield, 2003 & $\begin{array}{l}\text { ICPP-189, 198, 204, 205, 213, 214, } \\
215\end{array}$ & $\begin{array}{l}\text { Particle size, bulk density, particle density, and } \\
\text { specific surface area of sedimentary interbeds } \\
\text { from } 6 \text { cores from the vadose zone research } \\
\text { park near INTEC }\end{array}$ \\
\hline
\end{tabular}




\section{Appendixes}

Appendixes A and B are PDF files available for download at https://doi.org/10.3133/ofr20181001.

Appendix A. Idaho National Laboratory Lithologic Core Storage Library Standard Operating Procedures

Appendix B. Idaho National Laboratory Lithologic Core Storage Library Forms 
Publishing support provided by the U.S. Geological Survey Science Publishing Network, Tacoma Publishing Service Center

For more information concerning the research in this report, contact the Director, Idaho Water Science Center

U.S. Geological Survey

230 Collins Road

Boise, Idaho 83702

https://id.water.usgs.gov 
